\title{
Barreras geográficas de acceso a los servicios de salud oral en el departamento de Nariño, Colombia*
}

\section{Geographic Access Barriers to Oral Health Services in the Department of Nariño, Colombia}

\section{Barreiras geográficas de acesso aos serviços de saúde bucal, no departamento de Nariño, Colômbia}

Fecha de recepción: 11-05-16 Fecha de aceptación: 27-07-16 Disponible en línea: 30-11-16 doi:10.11144/Javeriana.rgyps15-31.bgas

Cómo citar este artículo:

Caicedo-Rosero AV, Estrada-Montoya JH. Barreras geográficas de acceso a los servicios de salud oral en el departamento de Nariño, Colombia. Rev. Gerenc. Polít. Salud. 2016; 15(31): 146-174. http://dx.doi. org/10.11144/Javeriana.rgyps15-31.bgas

\section{Ángela Viviana Caicedo-Rosero**} John Harold Estrada-Montoya***

Artículo de investigación.

Odontóloga, Estudiante de la especialización en Endodoncia, Universidad Nacional de Colombia. Bogotá, Colombia. Autor principal. Dirección postal: Universidad Nacional de Colombia, Carrera 30 No. 45-30, Edificio 210, Ciudad Universitaria, Bogotá, Colombia. Correo electrónico: avcaicedor@unal.edu.co Odontología, Departamento de Salud Colectiva, Universidad Nacional de Colombia, Bogotá, Colombia. Correo electrónico: jhestradam@unal.edu.co,o jhestradam@gmail.com 


\section{Resumen}

Estudio de corte trasversal cuyo objetivo fue identificar presencia de barreras geográficas de acceso a salud y elaborar un inventario de prestadores de salud oral del departamento de Nariño, con información de 64 municipios. Metodología: se desarrolló un sistema de medida de distancia y tiempo, identificando el tiempo de viaje que toma el desplazamiento de un municipio hasta la capital en diferentes medios de transporte. Como determinante de barrera de acceso: tiempo de viaje mayor a cuatro horas y distancia mayor a $25 \mathrm{~km}$. Resultados: se identificaron 305 prestadores públicos, 1062 privados; en salud oral 670 prestadores. Nariño cuenta con primer nivel de atención en todos los municipios, segundo en cuatro y tercero en Pasto. De los 64 municipios tres no tienen barrera de acceso. Conclusiones: el tiempo de viaje y el medio de transporte son determinantes de barrera de acceso a salud, especialmente en las áreas del Pacífico de Nariño.

Palabras clave: estructuras de acceso; accesibilidad a los servicios de salud; tiempo de viaje; ubicaciones geográficas; transporte de pacientes; disparidades en atención de salud

\section{Abstract}

A cross-sectional study aimed to identify the existence of geographic barriers to health care and to conduct an inventory of health care institutions in Nariño with information from the sixtyfour municipalities in the state. Methods: A measurement system was developed to identify the travel time from a municipality to Pasto in different means of transportation. A travel time of more than 4 hours or a distance longer than $25 \mathrm{~km}$ were considered access barriers. Results: Nariño has 305 providers in public sector, 1062 in private sector; 670 oral health providers was identify. Nariño has primary health care providers in the entire state, second level in four and third level only in Pasto. From the 64 municipalities analyzed, 3 did not have access barriers in terms of travel time or distance. Conclusions: The travel time and the means of transportation generate access barriers to health care services in Nariño, accentuated in pacific area.

Keywords: architectural accessibility; health services accessibility; travel; geography medical; geographic mapping; transportation of patients

\section{Resumo}

Estudo transversal teve como objetivo identificar a presença de barreiras geográficas ao acesso à saúde e desenvolver um inventário dos provedores de saúde departamento de Nariño oral, com informações de 64 municípios. Metodologia: Um sistema de medição foi desenvolvido distância e tempo, identificando o tempo de viagem leva o deslocamento de um município à capital em diferentes meios de transporte. Como determinante da barreira de acesso: mais tempo de viagem para quatro horas e superior a $25 \mathrm{~km}$ de distância. Resultados: 305 prestadores públicos, privados de 1062 foram identificados; 670 provedores de saúde bucal. Nariño tem primeiro nível de atenção em todos os municípios, quatro segundo e terceiro em Pasto. Dos 64 municípios de três não têm barreira de acesso. Conclusões: o tempo de viagem e os meios de transporte são o acesso barreira decisiva para a saúde, especialmente nas áreas de Nariño Pacífico.

Palavras-chave: estruturas de acesso; acessibilidade aos serviços de saúde; tempo de viagem; localizações geográficas; o transporte do paciente; disparidades nos cuidados de saúde 


\section{Introducción}

En Colombia, el Sistema General de Seguridad Social en Salud (SGSSS) no ha logrado los propósitos de cobertura universal y disminución de la inequidad, ni tampoco el mejoramiento significativo del acceso y la calidad de los servicios de salud. El papel preponderante dado al sector privado en el aseguramiento y la prestación de los servicios, los mecanismos de mercado y competencia (a lo que llamamos mercantilización de la salud), la desregulación y la desmejora de las condiciones laborales, entre otras medidas, no han dado los resultados esperados por quienes las propusieron como solución a la fragmentación existente antes de 1993. Por el contrario, a pesar del incremento en la cobertura de aseguramiento, la población debe superar múltiples barreras de acceso, relacionadas con los mecanismos de contención de costos, dado que la obtención de rentabilidad financiera está por encima de los criterios clínicos y de la necesidad de los usuarios - ya no pacientes-, como lo constatan las numerosas tutelas documentadas por la Defensoría del Pueblo que reflejan la violación reiterada del derecho a la salud.

La atención en salud en el país es costosa, por el proceso de intermediación y el afán de lucro de los múltiples actores privados (aseguradoras-EPS, clínicas privadas, farmacéuticas) en competencia y genera gastos adicionales a las personas, las cuales tienen que pagar cuotas mensuales y moderadoras o asumir de su bolsillo el pago de servicios. No es solidario, porque quienes ganan el mínimo o se contratan temporalmente contribuyen proporcionalmente más que quienes ganan mucho. La segmentación y la fragmentación del SGSSS son problemas que tienen efectos en la calidad, la equidad y el acceso, además del exceso de trámites administrativos que generan. Hay segmentación de la población por regímenes de afiliación, con planes de beneficio diferenciales por estrato socioeconómico.

La fragmentación estructural del SGSSS y la separación de servicios de promoción, prevención, tratamiento y rehabilitación se hace mayor en la contratación de servicios entre aseguradoras y entidades prestadoras. Los servicios que deben ser integrales, para atender enfermedades que ocurren en un mismo paciente, son separados y cada parte se contrata con entidades diferentes; por ejemplo, se contrata la atención de una patología con una institución, la hospitalización con otra y los insumos médicos con otra. Las barreras de acceso a salud son aquellas situaciones que limitan el acceso físico a los recursos de salud que pueden ser de diferentes tipos como sociales, culturales, normativas, administrativas, económicas y políticas; la ubicación geográfica es considerada una potencial barrera de acceso a los recursos (1-6).

Si se parte de la suposición de que en Colombia un ciudadano logra superar las barreras inherentes a la lógica neoliberal que opera en el sistema, y que configura múltiples itinerarios que se deben superar para acceder a los servicios, se encuentra que la geografía, entendida en esta investigación como la ubicación geográfica (en tiempo de viaje y en kilómetros) de los sitios de prestación de los servicios de salud general y oral, así como de la presencia o ausencia de profesionales odontólogos generales y/o con especializaciones clínicas en dichos sitios y la relación profesional-habitante, como se mencionó, se puede configurar como la primera barrera de acceso a los servicios de salud general y oral. ${ }^{1}$

1 Esta investigación asume que si dichos servicios cuentan con acreditación y habilitación de calidad, estarían en la capacidad de prestar el servicio demandado por el ciudadano, de acuerdo a su nivel de complejidad. Rebasa los alcances del trabajo, indagar por la idoneidad de las instalaciones y por las competencias de las personas que brindan los servicios, tema de investigaciones futuras. 
El sistema de salud de Colombia se rige por los principios de solidaridad, integralidad, universalidad y participación, y pretende estar disponible para toda la población sin barreras que lo limiten. Por tal razón, esta investigación buscó identificar si la distribución de los niveles de atención y el número de prestadores de salud y salud oral en un departamento -Nariño en este caso - son suficientes para atender a toda la población, e identificar si la distancia y el tiempo de viaje configuran una barrera de acceso al sistema de salud en este mismo departamento, teniendo en cuenta los distintos niveles de atención y las características de los servicios ofrecidos (7-10).

Con la Ley 100 de 1993 se logra el ordenamiento de la prestación pública de los servicios de salud a través de dos regímenes: el subsidiado y el contributivo, la forma de financiación y la organización del aseguramiento en entidades promotoras de salud (EPS), instituciones prestadoras de salud (IPS) y empresas sociales del Estado (ESE), las entidades de vigilancia y control, y se define que debe existir un plan obligatorio de salud (POS), que es más claro en la Resolución 5261 de 1994, la cual dispone las responsabilidades por niveles de atención (11-13):

- $\quad$ Nivel I: médico general y/o personal auxiliar y/o paramédico y/o de otros profesionales de la salud no especializados.

- Nivel II: médico general y/o profesional paramédico con interconsulta, remisión y/o asesoría de personal o recursos especializados.

- Niveles III y IV: médico especialista, con la participación del médico general y/o profesional paramédico.

El Estado debe garantizar a los ciudadanos los principios de la Ley 100, una cobertura amplia, de calidad, oportuna, continua y humana en todo el país; sin embargo, Colombia es un país con 32 departamentos, diversos culturalmente y dispersos geográficamente, y, además, con problemas de orden político y social que pueden limitar el desplazamiento libre dentro del país, con dificultades económicas, limitaciones en el transporte debido al estado o a la ausencia de vías de comunicación, lo que se convierte en un reto importante para el Estado y para lograr el objetivo de una cobertura universal y de calidad para los colombianos $(9,11,14)$.

El departamento de Nariño no escapa a esta realidad, por ello es importante conocer las dificultades para el acceso al sistema de salud, así como evidenciar la existencia o no de barreras de acceso a los servicios de salud debido a la geografía y a la disponibilidad de vías de comunicación en el departamento $(14,15)$.

La financiación de un sistema de salud se debe diseñar con base en proporcionar el acceso a los servicios sanitarios de calidad a toda la población, sin que ello implique dificultades económicas para el usuario. En el 2005 en la Asamblea Mundial de la Salud se instó a los países a que adoptaran sistemas de financiación que permitieran a toda la población el acceso a la salud, entendida esta desde todos sus aspectos: promoción, prevención, tratamiento y rehabilitación, sin una implicación económica para el usuario $(16,17)$.

El acceso a salud se refiere al acto de usar o recibir atención en salud, la búsqueda del servicio y la posibilidad de recibirlo. El acceso se piensa en términos de etapas y dimensiones. Se describen dos grandes etapas, según Guagliardo: el "potencial” de entrega de salud y la "realización" de la entrega de la salud. El potencial existe cuando una población coexiste en un espacio, un tiempo y un sistema de salud capaz de proveer la atención en salud. Y la realización se refiere al acceso a la salud cuando se superan todas 
las barreras. En el 2012 Waris Qidwai et al. (18) (definieron el acceso a la salud y a los cuidados de salud como la provisión y utilización de los servicios, e incluyeron también la disponibilidad del acceso a servicios y la facilidad para obtenerlos.

Es importante destacar que los conceptos de acceso y de accesibilidad involucran: acceso financiero (disponibilidad financiera de las personas para el acceso a salud); acceso conductual (utilización de los diversos servicios de salud); acceso espacial (relacionado con la distancia geográfica, el transporte y el tiempo de viaje). El acceso geográfico a la salud es la distancia entre un potencial consumidor y el proveedor del servicio, con cierto estándar de medida que involucra patrones de tráfico, medio de transporte y tiempo de viaje (cuatro horas o $25 \mathrm{~km})(18-21)$.

Estudios realizados en Canadá por Wilson y Rosenberg en el 2002 (22) encontraron que la accesibilidad geográfica a un sistema o proveedor de servicios de salud constituye una barrera de acceso; es decir, que no es suficiente con que el servicio de salud exista, hace falta que los usuarios tengan la posibilidad de llegar a él, en el lugar y el tiempo requeridos. Según Penchansky y Thomas, las barreras de acceso se agrupan en cinco dimensiones: disponibilidad, accesibilidad, asequibilidad, aceptabilidad y alojamiento (23).

Magilvy et al. (24) sostienen que en América la salud mejora, pero barreras como la distancia, la geografía y la pobre distribución de servicios y el cuidado de la salud hacen que el acceso se limite, especialmente para las personas de la tercera edad, quienes además de las barreras de acceso físicas que enfrentan, tienen limitaciones funcionales, escasos ingresos, alto riesgo de enfermedades, condiciones médicas de cuidado y limitaciones en el transporte que se suman a las demás barreras.
Los servicios de salud se concentran en las áreas urbanas y dejan descubierto el sector rural, lo cual hace que la población, en este último caso, no disfrute del mismo acceso a la salud que la población del área urbana. Cerca de la mitad de la población del mundo vive en áreas rurales, en condiciones de pobreza más acentuadas que en las áreas urbanas, con distancias más largas que recorrer hasta los sistemas de salud. Los recursos públicos de la salud dan soporte a las actividades diseñadas para promover la salud y prevenir la enfermedad, además del recurso profesional en diferentes áreas que pueda atender la mayoría de las necesidades de una población en el tiempo adecuado (24-26).

En el estudio de los servicios de salud deben ser consideradas tres dimensiones: capacitación del personal, predisposición a la enfermedad y necesidades de salud. En salud oral existe un recurso humano insuficiente en odontología para el desarrollo de las actividades clínicas de acción colectiva (27).

El desarrollo de estudios de tipo geográfico, que en un principio se encargaban de evaluar el impacto de la enfermedad y la gran relevancia que tenía en la distribución espacial y el curso de desarrollo de esta en un espacio determinado, motivó la elaboración de mapas que representaran los brotes de plagas, lo cual Koch llevó cabo primera vez en Italia. ciudad y ponían puntos o cruces por cada caso reportado, lo cual permitía reconocer zonas con mayor o menor prevalencia de casos de enfermos y/o muertos, y esto dio lugar a las primeras asociaciones causales con posibles fuentes de contaminación o transmisión, como pozos de agua o mercados de alimentos, entre otros. (28-30).

Los sistemas de información geográfica ofrecen información sobre la implementación de los sistemas de salud en las regiones y las estrategias de intervención de los programas de 
salud. La elaboración de mapas geográficos proporciona una visión clara de la variación en los fenómenos que afectan a la salud en su relación con la población y una geografía determinada. Generalmente, se puede ver cómo la geografía afecta a la enfermedad, a la salud y a los factores de riesgo presentes en una población, y asimismo permite la identificación del acceso a la atención en salud de una población determinada $(31,32)$.

\section{Materiales y métodos}

Estudio de corte transversal que tomó como muestra los 64 municipios del departamento de Nariño en Colombia, con una población total de 1498234 habitantes en el año 2005 (33).

La investigación se desarrolló en siete etapas, empezando en junio del 2011 y terminando en junio del 2013:

- Búsqueda de la bibliografía y selección de fuentes de información: las bases de datos consultadas fueron Pub Med, Jstor, Science DIrect y Medline. Las consultas se realizaron utilizando términos normalizados en $\mathrm{MeSH}$, cruzando todas ellas mediante el uso de operadores booleanos. Los términos usados fueron: geographical barriers, access to health, rural areas health, geography health.

- Recolección de datos: se consultó el Censo General 2005, Nivel Nacional del Departamento Administrativo Nacional de Estadística (DANE); se consultó el número de prestadores de salud y salud oral en el departamento de Nariño entre mayo y julio del 2012, en la base de datos de la página oficial del Ministerio de Salud y Protección Social. Se consultó en la Gobernación de Nariño, en el área de infraestructura, las vías de comunicación del departamento, su estado (afirmado o pavimentado), y en esta fase se obtuvieron los mapas actuales del departamento, con las vías de comunicación, actualizados al año 2013. En el Instituto Nacional de Vías (Invias) del departamento se obtuvo información, por medio de entrevista con funcionarios, acerca de las entidades encargadas de las vías, la distribución de los recursos y la regulación de las vías en los órdenes nacional, departamental y municipal $(14,33,34)$.

- Acercamiento interdisciplinario: se obtuvo asesoría de un técnico operativo de infraestructura y minas de la Gobernación de Nariño sobre la existencia o no de comunicación terrestre, el estado de las vías del departamento, los entes encargados de su manejo y la distribución del recurso para su mantenimiento, además de los mapas viales actualizados al año 2013. Un ingeniero electrónico se encargó de la asesoría en el manejo del programa SQL Server versión 2008, para el manejo de la base de datos y la organización de la información recogida por medio de filtros. La elaboración de mapas se logró con la asesoría de un ingeniero topográfico, quien con base en el mapa político del departamento de Nariño desarrolló, por medio de la aplicación a mano alzada del programa AutoCad e Inkscape, un mapa del departamento en capas que posteriormente fueron coloreados, según una escala de color elegida para datos numéricos (gamas de marrón y amarillo) y para barrera de acceso (gamas de azul).

- Desarrollo del sistema de medida de tiempo de viaje para la configuración como barrera de acceso: esto se logró mediante recorridos por las carreteras pavimentadas y afirmadas en los medios de transporte usados en el departamen- 
to: bicicleta, automóvil, caballo y a pie. Se realizaron recorridos de una hora; se tomó la hora inicial y final del recorrido y la distancia de desplazamiento en kilómetros. La distancia entre los municipios y la capital de departamento se obtuvo a partir de la información disponible en la página oficial de la Gobernación de Nariño y de los mapas de la red vial proporcionados por la Oficina de Infraestructura del departamento. Los cálculos se hicieron a partir de la fórmula básica de distancia cuando un cuerpo se mueve en aceleración uniformeme: $\mathrm{X}=\mathrm{V}^{*} \mathrm{t}$, donde $X$ es la distancia, $V$ la velocidad y $t$ el tiempo. De esta ecuación se despeja el tiempo, que es lo que nos interesa conocer, y queda la siguiente formula: $\mathrm{X} / \mathrm{V}=\mathrm{t}$.

- Consolidación de la información en bases de datos: una vez obtenida la información, se hizo una clasificación e integración de datos en bases datos de Excel 2010. Posteriormente, se hicieron consultas en SQL Server 2008 (programa de libre acceso), que es un software motor de base de datos, para conseguir los filtros de las bases de datos creadas en Excel, clasificar la información en rangos, obtener los filtros requeridos para la investigación y elaborar de este modo los inventarios de prestadores de salud y salud oral en el departamento de Nariño, inventario vial en Nariño e inventario de talento humano en salud oral (14).

- Análisis de la información para identificar las barreras de acceso: el análisis se basa en un caso de estudio realizado en el 2009 por la Organización Mundial de la Salud (OMS) en Malawi para determinar si existe o no barrera de acceso geográfica para el acceso a medicamentos antirretrovirales, donde se encontró que existe una barrera de acceso cuando el sujeto se encuentra a más de cuatro horas del centro de distribución más cercano. $\mathrm{Si}$, en cambio, vive dentro de un perímetro de cuatro horas de viaje para obtener los antirretrovirales, se considera que sí tiene posibilidad de acceso (6).

- Elaboración de mapas: con base en el mapa político del departamento de Nariño, se elaboraron mapas analíticos de la salud en el departamento. Para esto se vectorizaron el mapa de Nariño y los mapas municipales (archivos con extensión .jpg con baja resolución) con la herramienta a mano alzada de los programas Inkscape, Corel Draw y AutoCad, se importaron en un archivo tipo con extensión .svg, para posteriormente darles color según los resultados encontrados con la investigación en el primer programa mencionado.

\section{Resultados}

Organización territorial y estructura de los prestadores de salud en el departamento de Nariño

El departamento Nariño se encuentra ubicado al sur de Colombia. Su área es de $33265 \mathrm{~km}^{2}$, lo que corresponde al $2.9 \%$ del territorio del país. Limita al norte con el departamento del Cauca, al sur con Ecuador, al oriente con el departamento del Putumayo y al occidente con el Océano Pacífico. Su población es de 1498234 habitantes para el año 2005, de los cuales 706636 se encuentran en las cabeceras municipales y 794598 en el área rural. La división territorial del departamento es de 64 municipios, cuya capital es la ciudad de San Juan de Pasto.

Nariño tiene una geografía variada, con tres entornos geográficos principales: la llanura del Pacífico, que se caracteriza por altas 
temperaturas, lluvias abundantes y vegetación exuberante. La Región Andina, que se caracteriza por la penetración de la Cordillera de los Andes, que forma el llamado Nudo de los Pastos; a la vez tiene una penetración de la Cordillera Occidental, con formaciones volcánicas y una profunda depresión denominada Hoz de Miramá. Y la zona de la Cordillera Centro-Oriental, en donde se pueden encontrar altiplanos, valles, volcanes como el Galeras y el Doña Juana. En la vertiente amazónica se encuentra la laguna de La Cocha, zonas húmedas y terrenos poco aprovechables. El clima del departamento varía desde los $1.5^{\circ} \mathrm{C}$ hasta los $28^{\circ} \mathrm{C}$, y se encentran todos los pisos térmicos, desde los 2 msnm hasta los 4760 msnm (33-36).
La información demográfica de Nariño se contrasta con la información de los prestadores de salud en el departamento (figura 1 y tabla 1) obtenida de la página web oficial del Ministerio de Salud y la Protección Social, de la base de datos denominada Registro Actual, consultada en los meses de mayo y junio del 2012. Estos datos se consigna en la tabla 1, con las divisiones de prestadores públicos de acuerdo a los niveles de atención en salud: primer, segundo y tercer nivel de complejidad de atención en salud. Asimismo, el sector privado, el cual se subdivide en prestadores de baja, media y alta complejidad.

Se encontró que Nariño cuenta con los tres niveles de atención en salud del sector público, entre los cuales predomina el primer

Figura 1. Demografía del departamento de Nariño y ubicación de los PRESTAdores de SALUd de PRimer, SEGUNDO Y TERCER NIVEL DE ATENCIÓN

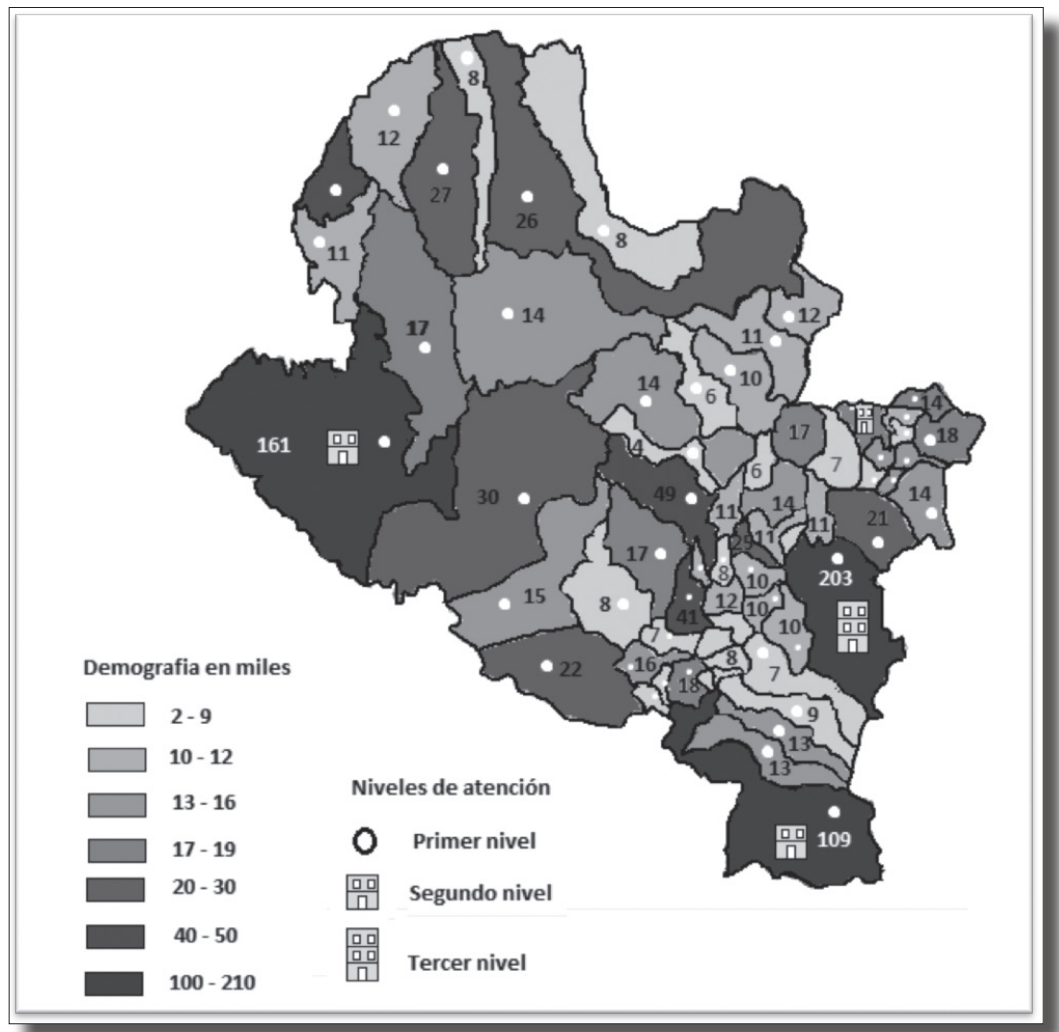

Fuente: elaboración propia 
nivel, que cubre al $100 \%$ de los municipios, el segundo nivel se encuentra en cuatro municipios: Pasto, Ipiales, Tumaco y La Unión, y el nivel tres solo en Pasto. En cuanto al sector privado de atención en salud, es evidente que la fuerza laboral se concentra en la ciudad de Pasto, en todos los niveles de complejidad, seguida de Ipiales, Tumaco y La Unión, con predominio del nivel de atención de baja complejidad.

Tabla 1. Demografía del dePartamento de Nariño y distribución de los niveles de atención Pública y PRIVADA EN CADA MUNICIPIO

\begin{tabular}{|c|c|c|c|c|c|c|c|c|c|}
\hline & \multicolumn{3}{|c|}{ Demografía } & \multicolumn{6}{|c|}{ Nivel de atención } \\
\hline & \multirow[b]{2}{*}{ Hombres } & \multirow[b]{2}{*}{ Mujeres } & \multirow[b]{2}{*}{ Total } & \multicolumn{3}{|c|}{ Público } & \multicolumn{3}{|c|}{ Privado } \\
\hline & & & & Primero & Segundo & Tercero & $\begin{array}{l}\text { Compleji- } \\
\text { dad baja }\end{array}$ & $\begin{array}{l}\text { Compleji- } \\
\text { dad media }\end{array}$ & $\begin{array}{l}\text { Compleji- } \\
\text { dad alta }\end{array}$ \\
\hline Nariño & 741925 & 756309 & 1498234 & 300 & 4 & 1 & 698 & 330 & 34 \\
\hline Pasto & 383846 & 181434 & 202412 & 28 & 1 & 1 & 376 & 246 & 29 \\
\hline Albán & 9987 & 9380 & 19367 & 5 & 0 & 0 & 0 & 0 & 0 \\
\hline Aldana & 3270 & 3510 & 6780 & 2 & 0 & 0 & 0 & 0 & 0 \\
\hline Ancuyá & 4247 & 4057 & 8304 & 1 & 0 & 0 & 2 & 0 & 0 \\
\hline Arboleda & 3837 & 3605 & 7442 & 3 & 0 & 0 & 0 & 0 & 0 \\
\hline Barbacoas & 15268 & 14988 & 30256 & 18 & 0 & 0 & 3 & 0 & 0 \\
\hline Belén & 2436 & 2489 & 4925 & 2 & 0 & 0 & 0 & 0 & 0 \\
\hline Buesaco & 10523 & 10496 & 21019 & 8 & 0 & 0 & 7 & 0 & 0 \\
\hline Chachagüí & 6048 & 5862 & 11910 & 5 & 0 & 0 & 1 & 0 & 0 \\
\hline Colón & 5017 & 4655 & 9672 & 2 & 0 & 0 & 2 & 0 & 0 \\
\hline Consaca & 5062 & 5147 & 10209 & 1 & 0 & 0 & 0 & 1 & 0 \\
\hline Contadero & 3330 & 3309 & 6639 & 1 & 0 & 0 & 0 & 0 & 0 \\
\hline Córdoba & 6558 & 6905 & 13463 & 6 & 0 & 0 & 4 & 0 & 0 \\
\hline Cuaspud & 3987 & 4121 & 8108 & 2 & 0 & 0 & 1 & 0 & 0 \\
\hline Cumbal & 11146 & 11272 & 22418 & 14 & 0 & 0 & 6 & 1 & 0 \\
\hline Cumbitara & 3142 & 3000 & 6142 & 1 & 0 & 0 & 0 & 0 & 0 \\
\hline El charco & 13193 & 12970 & 26163 & 9 & 0 & 0 & 1 & 0 & 0 \\
\hline El Peñol & 3468 & 3215 & 6683 & 1 & 0 & 0 & 1 & 0 & 0 \\
\hline El Rosario & 5953 & 5251 & 11204 & 5 & 0 & 0 & 0 & 0 & 0 \\
\hline $\begin{array}{l}\text { El Tablón } \\
\text { de Gómez }\end{array}$ & 7106 & 6784 & 13890 & 3 & 0 & 0 & 1 & 0 & 0 \\
\hline El tambo & 6896 & 6903 & 13799 & 3 & 0 & 0 & 4 & 0 & 0 \\
\hline $\begin{array}{l}\text { Francisco } \\
\text { Pizarro }\end{array}$ & 6002 & 5181 & 11183 & 5 & 0 & 0 & 0 & 0 & 0 \\
\hline Funes & 3474 & 3213 & 6687 & 1 & 0 & 0 & 0 & 0 & 0 \\
\hline Guachucal & 8173 & 8454 & 16627 & 3 & 0 & 0 & 7 & 2 & 0 \\
\hline Guaitarilla & 6416 & 6348 & 12764 & 1 & 0 & 0 & 4 & 0 & 0 \\
\hline Gualmatán & 2681 & 2975 & 5656 & 1 & 0 & 0 & 2 & 0 & 0 \\
\hline Iles & 3940 & 3927 & 7867 & 1 & 0 & 0 & 0 & 0 & 0 \\
\hline Imués & 3706 & 3681 & 7387 & 2 & 0 & 0 & 0 & 0 & 0 \\
\hline Ipiales & 53057 & 56808 & 109865 & 18 & 1 & 0 & 104 & 38 & 3 \\
\hline La Cruz & 8966 & 8667 & 17633 & 7 & 0 & 0 & 11 & 1 & 0 \\
\hline
\end{tabular}




\begin{tabular}{|c|c|c|c|c|c|c|c|c|c|}
\hline & \multicolumn{3}{|c|}{ Demografía } & \multicolumn{6}{|c|}{ Nivel de atención } \\
\hline & \multirow[b]{2}{*}{ Hombres } & \multirow[b]{2}{*}{ Mujeres } & \multirow[b]{2}{*}{ Total } & \multicolumn{3}{|c|}{ Público } & \multicolumn{3}{|c|}{ Privado } \\
\hline & & & & Primero & Segundo & Tercero & $\begin{array}{l}\text { Compleji- } \\
\text { dad baja }\end{array}$ & $\begin{array}{l}\text { Compleji- } \\
\text { dad media }\end{array}$ & $\begin{array}{l}\text { Compleji- } \\
\text { dad alta }\end{array}$ \\
\hline La florida & 5551 & 5600 & 11151 & 2 & 0 & 0 & 1 & 0 & 0 \\
\hline La Llanada & 1918 & 1776 & 3694 & 1 & 0 & 0 & 0 & 0 & 0 \\
\hline La tola & 4524 & 4047 & 8571 & 1 & 0 & 0 & 0 & 0 & 0 \\
\hline La unión & 13913 & 13675 & 27588 & 6 & 1 & 0 & 17 & 6 & 0 \\
\hline Leiva & 6174 & 5651 & 11825 & 1 & 0 & 0 & 2 & 0 & 0 \\
\hline Linares & 5908 & 5638 & 11546 & 2 & 0 & 0 & 0 & 0 & 0 \\
\hline Los andes & 7540 & 7330 & 14870 & 1 & 0 & 0 & 2 & 0 & 0 \\
\hline Magüi & 7246 & 6585 & 13831 & 12 & 0 & 0 & 0 & 0 & 0 \\
\hline Mallama & 4210 & 4107 & 8317 & 1 & 0 & 0 & 4 & 0 & 0 \\
\hline Mosquera & 6042 & 5831 & 11873 & 1 & 0 & 0 & 0 & 0 & 0 \\
\hline Nariño & 2056 & 2154 & 4210 & 1 & 0 & 0 & 1 & 0 & 0 \\
\hline $\begin{array}{l}\text { Olaya } \\
\text { herrera }\end{array}$ & 14215 & 13010 & 27225 & 7 & 0 & 0 & 2 & 0 & 0 \\
\hline Ospina & 4212 & 4021 & 8233 & 1 & 0 & 0 & 0 & 0 & 0 \\
\hline Policarpa & 5090 & 4708 & 9798 & 1 & 0 & 0 & 1 & 1 & 0 \\
\hline Potosí & 6535 & 6505 & 13040 & 15 & 0 & 0 & 0 & 0 & 0 \\
\hline Providencia & 5777 & 5949 & 11726 & 1 & 0 & 0 & 0 & 0 & 0 \\
\hline Puerres & 4271 & 4579 & 8850 & 6 & 0 & 0 & 1 & 0 & 0 \\
\hline Pupiales & 8991 & 9424 & 18415 & 2 & 0 & 0 & 5 & 0 & 0 \\
\hline Ricaurte & 7558 & 7111 & 14669 & 2 & 0 & 0 & 5 & 0 & 0 \\
\hline $\begin{array}{l}\text { Roberto } \\
\text { payán }\end{array}$ & 8912 & 8374 & 17286 & 8 & 0 & 0 & 0 & 0 & 0 \\
\hline Samaniego & 25142 & 24850 & 49992 & 1 & 0 & 0 & 10 & 1 & 0 \\
\hline $\begin{array}{l}\text { San } \\
\text { bernardo }\end{array}$ & 7533 & 6954 & 14487 & 1 & 0 & 0 & 0 & 0 & 0 \\
\hline $\begin{array}{l}\text { San } \\
\text { lorenzo }\end{array}$ & 9551 & 8879 & 18430 & 3 & 0 & 0 & 0 & 0 & 0 \\
\hline San pablo & 7360 & 7572 & 14932 & 2 & 0 & 0 & 2 & 0 & 0 \\
\hline $\begin{array}{l}\text { San Pedro } \\
\text { de Cartago }\end{array}$ & 3643 & 3404 & 7047 & 1 & 0 & 0 & 0 & 0 & 0 \\
\hline Sandoná & 12979 & 12155 & 25134 & 5 & 0 & 0 & 14 & 0 & 0 \\
\hline $\begin{array}{l}\text { Santa } \\
\text { bárbara }\end{array}$ & 4501 & 4114 & 8615 & 2 & 0 & 0 & 2 & 1 & 0 \\
\hline Santacruz & 8456 & 8413 & 16869 & 7 & 0 & 0 & 0 & 0 & 0 \\
\hline Sapuyes & 3618 & 3751 & 7369 & 3 & 0 & 0 & 2 & 0 & 0 \\
\hline Taminango & 8806 & 8548 & 17354 & 1 & 0 & 0 & 6 & 0 & 0 \\
\hline Tangua & 5363 & 5309 & 10672 & 1 & 0 & 0 & 1 & 0 & 0 \\
\hline Tumaco & 80815 & 80675 & 161490 & 39 & 1 & 0 & 55 & 25 & 2 \\
\hline Túquerres & 20260 & 20945 & 41205 & 3 & 0 & 0 & 27 & 7 & 0 \\
\hline Yacuanquer & 4932 & 5080 & 10012 & 1 & 0 & 0 & 1 & 0 & 0 \\
\hline
\end{tabular}

Fuente:DANEy Registro Especial de Prestadores de Salud Actual(REPS) del Ministerio de Saludy la Protección Social 
En cuanto a los niveles de atención en salud del sector público, se evidenció que los 64 municipios tienen acceso al menos a un centro de salud, que comprende la atención por un médico general, odontología general, atención de urgencias, atención prioritaria, promoción y prevención, personal auxiliar y laboratorio. El primer nivel de atención en salud es predominante en el municipio de Tumaco, con 39 prestadores de salud, seguido por el municipio de Pasto con 28, de un total departamental de 300 prestadores en el sector público.

La distribución de los prestadores de salud en el departamento se concentra en la ciudad de Pasto. El segundo nivel de atención en salud es limitado y se presenta como una barrera de acceso a los demás municipios, ya que se concentra en solo cuatro: Pasto, Ipiales, La Unión y Tumaco. En cada uno de estos municipios solo se encontró un centro de atención de segundo nivel y los sesenta municipios restantes no cuentan con este nivel de atención del sector público en salud. Por lo tanto, al momento de requerir atención de tipo hospitalario, o con recursos especializados, la población debe desplazarse hasta el municipio más cercano que cuente con el nivel de atención que se requiere. En la tabla 2 se recoge esta concentración de los prestadores de salud del segundo nivel de complejidad del sector privado. El tercer nivel de atención se limita al municipio de San Juan de Pasto, con solamente un hospital de tercer nivel. Esto representa que las personas del resto del departamento que requieren esta atención deben desplazarse hasta la capital para poder tener acceso, lo cual se dificulta por la distancia y la geografía del departamento.

En cuanto al sector privado de atención en salud, se encontró que en el departamento predomina el nivel de atención de baja complejidad (698 prestadores). Este comprende la misma complejidad del primer nivel del sector público. Estos prestadores se concentran en la ciudad de Pasto, con un total de 376, seguida por Ipiales con 104 y Tumaco con 55. Es importante destacar que hay municipios donde no se encuentran prestadores del sector privado de ningún nivel (25 municipios de los 64 del departamento). El nivel de media complejidad en salud del sector privado comprende los servicios similares a los del segundo nivel de atención en salud del sector público, pero se encuentran en una mayor proporción. Hay municipios que no tienen segundo nivel de complejidad (público), pero sí cuentan con la complejidad media por parte del sector privado; sin embargo, no se logra cubrir todas las necesidades de una población, dado que no se contemplan todos los servicios unificados del segundo nivel (público). Es evidente que el personal profesional de este

Tabla 2. Municipios con Segundo nivel de complejidad en el Departamento de Nariño, 2012

\begin{tabular}{|l|c|c|}
\hline \multicolumn{3}{|c|}{ Segundo nivel de atención } \\
\hline Municipio & Número de prestadores & Porcentaje \\
\hline Departamento & 4 & 100 \\
\hline Pasto & 1 & 25 \\
\hline Ipiales & 1 & 25 \\
\hline La Unión & 1 & 25 \\
\hline Tumaco & 1 & 25 \\
\hline Resto del departamento & 0 & 0 \\
\hline
\end{tabular}

Fuente: Ministerio de Salud y la Protección Social 
nivel se concentra en la ciudad de Pasto, en un $74 \%$, y en Ipiales, con un $11 \%$ del total del departamento, y el restante $15 \%$ de los prestadores está distribuido en 10 municipios; 52 municipios no cuentan con este nivel de complejidad (tabla 3).

En el departamento de Nariño, en cuanto al nivel de complejidad alta del sector privado, se encuentran 34 prestadores y persiste la concentración de estos en la ciudad de Pasto, con un total de 29, seguida por Ipiales con 3 y Tumaco con 2; el resto del departamento no cuenta con este nivel de complejidad. Asimismo, el sector privado es más amplio en número de prestadores que el sector público, que solo cuenta con uno en todo el departamento.

\section{Recursos humanos en salud oral}

En el estudio realizado por la Dirección del Talento Humano en Salud en el 2012, se encontró que en el país se cuenta con: auxiliares de salud oral, mayoritariamente mujeres, con una proporción con respecto a los hombres de 1:15; tecnólogos en mecánica dental, con una proporción de 2.5:1 de mujeres con respecto a hombres, y con un total de 14181 odontólogos. La odontología es la tercera profesión en salud más desarrollada en Colombia, después de la medicina y la enfermería, respectivamente (37). Los datos correspondientes al recurso humano en salud oral en el departamento de Nariño están registrados en la tabla 4 .

No todos los municipios del departamento tienen la misma condición de recursos en salud oral, ya que se encontraron $17 \mathrm{mu}$ nicipios que tienen de 4 a 6 prestadores de salud oral, lo cual se considera un bajo número de prestadores para atender a la población y demuestra que los servicios de salud oral se prestan con disponibilidad baja y no pueden responder de forma adecuada a las necesidades de salud oral de la población. La figura 2 permite observar de forma rápida y sencilla que el número de prestadores de salud oral en Nariño es bajo con respecto a la distribución territorial y a la demografía.

TABla 3. Diez PRIMERos MUNiCIPIOS CON MAYOR NÚMERO DE PRESTADORES DE MEDIA COMPLEJIDAD EN EL SECTOR PRIVADO EN EL DEPARTAMENTO DE NARIÑO, 2012

\begin{tabular}{|l|c|c|}
\hline \multicolumn{3}{|c|}{ Media complejidad de atención en salud en el sector privado } \\
\hline Municipio & Número de prestadores & Porcentaje \\
\hline Departamento & 330 & 100 \\
\hline Pasto & 246 & 74.55 \\
\hline Ipiales & 38 & 11.52 \\
\hline Tumaco & 25 & 7.58 \\
\hline Túquerres & 7 & 2.12 \\
\hline La Unión & 6 & 1.82 \\
\hline Guachucal & 2 & 0.61 \\
\hline Consaca & 1 & 0.30 \\
\hline Cumbal & 1 & 0.30 \\
\hline La Cruz & 1 & 0.30 \\
\hline Policarpa & 1 & 0.30 \\
\hline Resto del departamento & 2 & 0.61 \\
\hline
\end{tabular}

Fuente: Ministerio de Salud y la Protección Social 
Tabla 4. InVEntario de Recursos humanos en SAlud oral en El Departamento de Nariño, 2012

\begin{tabular}{|c|c|c|c|c|c|c|c|c|c|c|c|}
\hline \multirow[b]{2}{*}{ Municipio } & \multicolumn{8}{|c|}{ Especialidades } & \multicolumn{2}{|c|}{$\begin{array}{l}\text { Odontología } \\
\text { general }\end{array}$} & \multirow{2}{*}{$\begin{array}{c}\text { Total } \\
\text { especia- } \\
\text { lidades } \\
\text { y odon- } \\
\text { tología } \\
\text { general }\end{array}$} \\
\hline & $\begin{array}{c}\text { En- } \\
\text { dodon- } \\
\text { cia }\end{array}$ & $\begin{array}{c}\text { Ci- } \\
\text { rugía } \\
\text { maxi- } \\
\text { lofa- } \\
\text { cial }\end{array}$ & $\begin{array}{c}\text { Ci- } \\
\text { rugía } \\
\text { oral }\end{array}$ & $\begin{array}{c}\text { Orto- } \\
\text { doncia }\end{array}$ & $\begin{array}{l}\text { Perio- } \\
\text { doncia }\end{array}$ & $\begin{array}{c}\text { Odon- } \\
\text { tope- } \\
\text { diatría }\end{array}$ & $\begin{array}{c}\text { Reha- } \\
\text { bilita- } \\
\text { ción } \\
\text { oral }\end{array}$ & $\begin{array}{l}\text { Total } \\
\text { espe- } \\
\text { cialis- } \\
\text { tas }\end{array}$ & $\begin{array}{l}\text { Pú- } \\
\text { blico }\end{array}$ & $\begin{array}{l}\text { Pri- } \\
\text { vado }\end{array}$ & \\
\hline Nariño & 26 & 11 & 14 & 63 & 15 & 20 & 21 & 174 & 224 & 276 & 674 \\
\hline Pasto & 22 & 11 & 13 & 40 & 15 & 20 & 18 & 139 & 29 & 143 & 311 \\
\hline Albán & 0 & 0 & 0 & 0 & 0 & 0 & 0 & 0 & 5 & 1 & 6 \\
\hline Aldana & 0 & 0 & 0 & 0 & 0 & 0 & 0 & 0 & 2 & 0 & 2 \\
\hline Ancuyá & 0 & 0 & 0 & 0 & 0 & 0 & 0 & 0 & 2 & 0 & 2 \\
\hline Arboleda & 0 & 0 & 0 & 0 & 0 & 0 & 0 & 0 & 1 & 0 & 1 \\
\hline Barbacoas & 0 & 0 & 0 & 0 & 0 & 0 & 0 & 0 & 17 & 0 & 17 \\
\hline Belén & 0 & 0 & 0 & 0 & 0 & 0 & 0 & 0 & 1 & 0 & 1 \\
\hline Buesaco & 0 & 0 & 0 & 0 & 0 & 0 & 0 & 0 & 2 & 1 & 3 \\
\hline $\begin{array}{l}\text { Colon } \\
\text { (Genova) }\end{array}$ & 0 & 0 & 0 & 0 & 0 & 0 & 0 & 0 & 1 & 3 & 4 \\
\hline Consaca & 0 & 0 & 0 & 1 & 0 & 0 & 0 & 1 & 1 & 0 & 2 \\
\hline Contadero & 0 & 0 & 0 & 0 & 0 & 0 & 0 & 0 & 1 & 0 & 1 \\
\hline Córdoba & 0 & 0 & 0 & 0 & 0 & 0 & 0 & 0 & 2 & 1 & 3 \\
\hline Cuaspud & 0 & 0 & 0 & 0 & 0 & 0 & 0 & 0 & 2 & 0 & 2 \\
\hline Cumbal & 0 & 0 & 0 & 1 & 0 & 0 & 0 & 1 & 14 & 4 & 19 \\
\hline Cumbitara & 0 & 0 & 0 & 0 & 0 & 0 & 0 & 0 & 1 & 0 & 1 \\
\hline Chachagüi & 0 & 0 & 0 & 0 & 0 & 0 & 0 & 0 & 4 & 0 & 4 \\
\hline El Charco & 0 & 0 & 0 & 0 & 0 & 0 & 0 & 0 & 1 & 0 & 1 \\
\hline El Peñol & 0 & 0 & 0 & 0 & 0 & 0 & 0 & 0 & 1 & 0 & 1 \\
\hline El Rosario & 0 & 0 & 0 & 0 & 0 & 0 & 0 & 0 & 3 & 0 & 3 \\
\hline $\begin{array}{l}\text { El Tablón } \\
\text { de Gómez }\end{array}$ & 0 & 0 & 0 & 0 & 0 & 0 & 0 & 0 & 3 & 0 & 3 \\
\hline El Tambo & 0 & 0 & 0 & 0 & 0 & 0 & 0 & 0 & 1 & 1 & 2 \\
\hline Funes & 0 & 0 & 0 & 0 & 0 & 0 & 0 & 0 & 1 & 0 & 1 \\
\hline Guachucal & 0 & 0 & 0 & 1 & 0 & 0 & 0 & 1 & 3 & 4 & 8 \\
\hline Guaitarilla & 0 & 0 & 0 & 1 & 0 & 0 & 0 & 1 & 1 & 2 & 4 \\
\hline Gualmatán & 0 & 0 & 0 & 0 & 0 & 0 & 0 & 0 & 1 & 2 & 3 \\
\hline Iles & 0 & 0 & 0 & 0 & 0 & 0 & 0 & 0 & 1 & 0 & 1 \\
\hline Imués & 0 & 0 & 0 & 0 & 0 & 0 & 0 & 0 & 1 & 0 & 1 \\
\hline Ipiales & 3 & 0 & 1 & 11 & 0 & 0 & 1 & 16 & 18 & 50 & 84 \\
\hline La Cruz & 0 & 0 & 0 & 1 & 0 & 0 & 0 & 1 & 1 & 5 & 7 \\
\hline La Florida & 0 & 0 & 0 & 0 & 0 & 0 & 0 & 0 & 1 & 0 & 1 \\
\hline La Llanada & 0 & 0 & 0 & 0 & 0 & 0 & 0 & 0 & 1 & 0 & 1 \\
\hline La Tola & 0 & 0 & 0 & 0 & 0 & 0 & 0 & 0 & 4 & 0 & 4 \\
\hline La Unión & 0 & 0 & 0 & 2 & 0 & 0 & 1 & 3 & 3 & 8 & 14 \\
\hline Leiva & 0 & 0 & 0 & 0 & 0 & 0 & 0 & 0 & 1 & 1 & 2 \\
\hline
\end{tabular}




\begin{tabular}{|c|c|c|c|c|c|c|c|c|c|c|c|}
\hline & & & & Especi & lidades & & & & $\begin{array}{r}\text { Odon } \\
\text { gen }\end{array}$ & $\begin{array}{l}\text { logía } \\
\text { ral }\end{array}$ & \\
\hline Municipio & $\begin{array}{c}\text { En- } \\
\text { dodon- } \\
\text { cia }\end{array}$ & $\begin{array}{c}\text { Ci- } \\
\text { rugía } \\
\text { maxi- } \\
\text { lofa- } \\
\text { cial }\end{array}$ & $\begin{array}{l}\text { Ci- } \\
\text { rugía } \\
\text { oral }\end{array}$ & $\begin{array}{c}\text { Orto- } \\
\text { doncia }\end{array}$ & $\begin{array}{c}\text { Perio- } \\
\text { doncia }\end{array}$ & $\begin{array}{c}\text { Odon- } \\
\text { tope- } \\
\text { diatría }\end{array}$ & $\begin{array}{c}\text { Reha- } \\
\text { bilita- } \\
\text { ción } \\
\text { oral }\end{array}$ & $\begin{array}{l}\text { Total } \\
\text { espe- } \\
\text { cialis- } \\
\text { tas }\end{array}$ & $\begin{array}{c}\text { Pú- } \\
\text { blico }\end{array}$ & $\begin{array}{l}\text { Pri- } \\
\text { vado }\end{array}$ & $\begin{array}{l}\text { lidades } \\
\text { y odon- } \\
\text { tología } \\
\text { general }\end{array}$ \\
\hline Linares & 0 & 0 & 0 & 0 & 0 & 0 & 0 & 0 & 2 & 0 & 2 \\
\hline Los Andes & 0 & 0 & 0 & 0 & 0 & 0 & 0 & 0 & 1 & 1 & 2 \\
\hline Magüi & 0 & 0 & 0 & 0 & 0 & 0 & 0 & 0 & 5 & 1 & 6 \\
\hline Mallama & 0 & 0 & 0 & 0 & 0 & 0 & 0 & 0 & 1 & 1 & 2 \\
\hline Mosquera & 0 & 0 & 0 & 0 & 0 & 0 & 0 & 0 & 1 & 0 & 1 \\
\hline Nariño & 0 & 0 & 0 & 0 & 0 & 0 & 0 & 0 & 1 & 0 & 1 \\
\hline $\begin{array}{l}\text { Olaya } \\
\text { Herrera }\end{array}$ & 0 & 0 & 0 & 0 & 0 & 0 & 0 & 0 & 7 & 0 & 7 \\
\hline Ospina & 0 & 0 & 0 & 0 & 0 & 0 & 0 & 0 & 1 & 0 & 1 \\
\hline $\begin{array}{l}\text { Francisco } \\
\text { Pizarro }\end{array}$ & 0 & 0 & 0 & 0 & 0 & 0 & 0 & 0 & 4 & 0 & 4 \\
\hline Policarpa & 0 & 0 & 0 & 0 & 0 & 0 & 0 & 0 & 1 & 1 & 2 \\
\hline Potosí & 0 & 0 & 0 & 0 & 0 & 0 & 0 & 0 & 1 & 0 & 1 \\
\hline $\begin{array}{l}\text { Providen- } \\
\text { cia }\end{array}$ & 0 & 0 & 0 & 0 & 0 & 0 & 0 & 0 & 1 & 0 & 1 \\
\hline Puerres & 0 & 0 & 0 & 0 & 0 & 0 & 0 & 0 & 1 & 2 & 3 \\
\hline Pupiales & 0 & 0 & 0 & 0 & 0 & 0 & 0 & 0 & 2 & 5 & 7 \\
\hline Ricaurte & 1 & 0 & 0 & 1 & 1 & 0 & 1 & 0 & 1 & 3 & 8 \\
\hline $\begin{array}{l}\text { Roberto } \\
\text { Payán }\end{array}$ & 0 & 0 & 0 & 0 & 0 & 0 & 0 & 0 & 5 & 0 & 5 \\
\hline Samaniego & 0 & 0 & 0 & 0 & 0 & 0 & 0 & 0 & 3 & 2 & 5 \\
\hline Sandoná & 0 & 0 & 0 & 0 & 0 & 0 & 0 & 0 & 1 & 6 & 7 \\
\hline $\begin{array}{l}\text { San } \\
\text { Bernardo }\end{array}$ & 0 & 0 & 0 & 0 & 0 & 0 & 0 & 0 & 1 & 0 & 1 \\
\hline $\begin{array}{l}\text { San } \\
\text { Lorenzo }\end{array}$ & 0 & 0 & 0 & 0 & 0 & 0 & 0 & 0 & 1 & 0 & 1 \\
\hline San Pablo & 0 & 0 & 0 & 1 & 0 & 0 & 0 & 1 & 2 & 3 & 6 \\
\hline $\begin{array}{l}\text { San Pedro } \\
\text { de Cartago }\end{array}$ & 0 & 0 & 0 & 0 & 0 & 0 & 0 & 0 & 1 & 0 & 1 \\
\hline $\begin{array}{l}\text { Santa } \\
\text { Bárbara }\end{array}$ & 0 & 0 & 0 & 0 & 0 & 0 & 0 & 0 & 1 & 0 & 1 \\
\hline Santacruz & 0 & 0 & 0 & 0 & 0 & 0 & 0 & 0 & 2 & 0 & 2 \\
\hline Sapuyes & 0 & 0 & 0 & 0 & 0 & 0 & 0 & 0 & 2 & 0 & 2 \\
\hline Taminango & 0 & 0 & 0 & 0 & 0 & 0 & 0 & 0 & 3 & 3 & 6 \\
\hline Tangua & 0 & 0 & 0 & 0 & 0 & 0 & 0 & 0 & 1 & 1 & 2 \\
\hline Tumaco & 1 & 0 & 0 & 3 & 0 & 0 & 1 & 5 & 36 & 21 & 62 \\
\hline Túquerres & 0 & 0 & 0 & 1 & 0 & 0 & 0 & 1 & 3 & 0 & 4 \\
\hline $\begin{array}{l}\text { Yacuan- } \\
\text { quer }\end{array}$ & 0 & 0 & 0 & 0 & 0 & 0 & 0 & 0 & 1 & 0 & 1 \\
\hline
\end{tabular}

Fuente: Ministerio de Salud y la Protección Social 
Figura 2. Distribución de los prestadores de salud oral en el departamento de Nariño, 2012

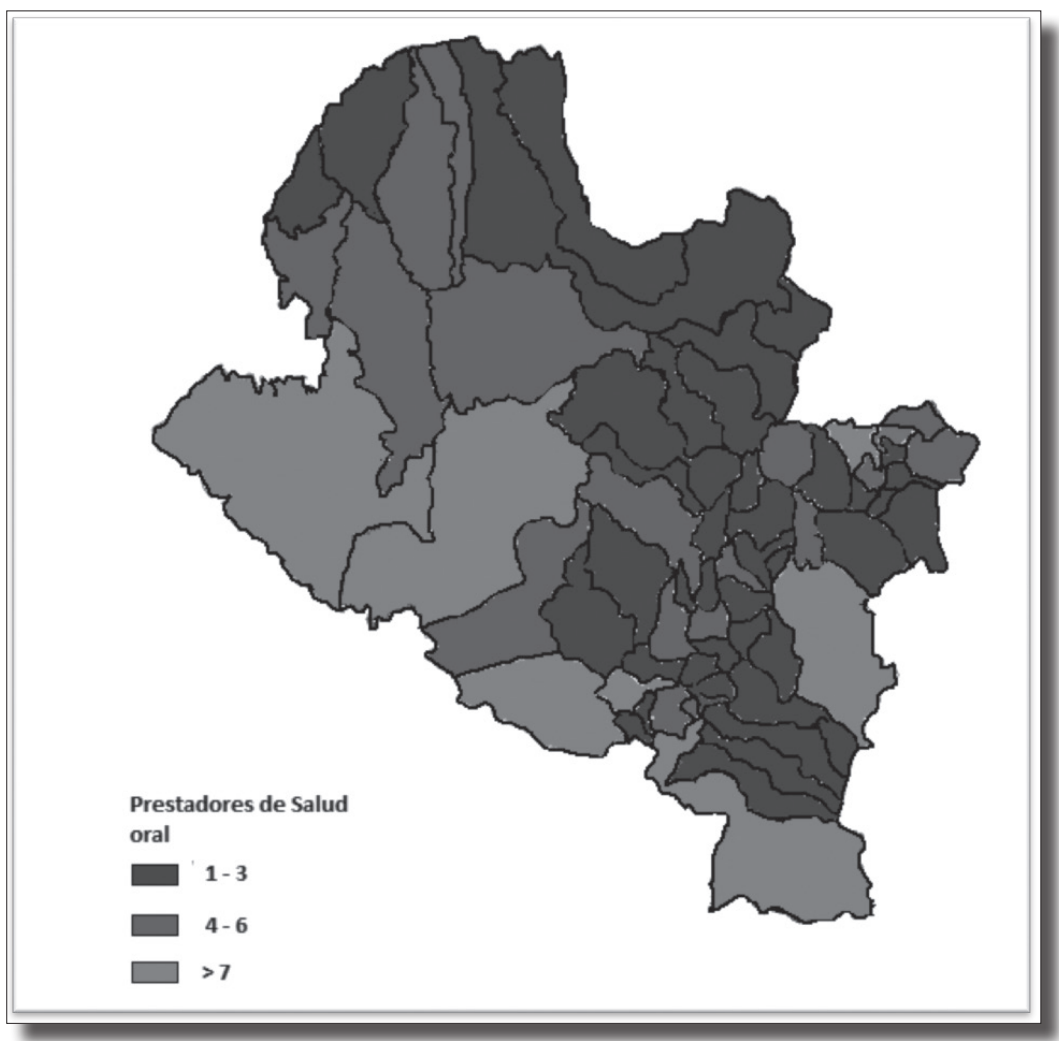

Fuente: elaboración propia

El departamento cuenta con una cobertura del $100 \%$ de odontología general por parte del sector público, es decir, que todos los municipios del departamento cuentan con al menos un odontólogo general. El total del departamento corresponde a 224 prestadores, con una mayor presencia en número en el municipio de Tumaco, donde se concentra el $16.1 \%$ de los prestadores del servicio; en Pasto el $13 \%$ de los prestadores y en Ipiales el $8 \%$. El número y la distribución territorial de los prestadores de salud oral se encuentran en la tabla 5.

Estos datos pueden llevar al supuesto de que la cobertura por odontología general del sector público es universal y no se presenta con una barrera de acceso geográfica en el departamento de Nariño, pero para ello es necesario identificar la disponibilidad del servicio, la oferta y la demanda de este.

En cuanto al sector privado, la odontología general logra un cubrimiento del 59\% de los municipios de Nariño, con un total de 276 prestadores del servicio de baja complejidad. El talento humano se concentra en un gran porcentaje en la ciudad de Pasto, correspondiente al $51 \%$ del total de los prestadores, con una amplia diferencia con respecto al resto del departamento, puesto que el siguiente municipio con mayor número de prestadores es Ipiales, con el $18 \%$ del total departamental, seguido de Tumaco con el 7.6\%. Esto hace evidente que si bien el sector privado de la odontología general tiene un número mayor de prestadores, no se encuentra en la capacidad de atender a toda la población del departamento, debido a 
Tabla 5. Número de PRestadores de odontología general En El dePartamento de Nariño, SECTOR PÚBLICO, 2012

\begin{tabular}{|l|c|c|}
\hline \multicolumn{3}{|c|}{ Odontología general (público) } \\
\hline Naturaleza jurídica & Público & Porcentaje \\
\hline Departamento & 224 & 100 \\
\hline Tumaco & 36 & 16.1 \\
\hline Pasto & 29 & 12.9 \\
\hline Ipiales & 18 & 8.0 \\
\hline Barbacoas & 17 & 7.6 \\
\hline Cumbal & 14 & 6.3 \\
\hline Olaya Herrera & 7 & 3.1 \\
\hline Albán & 5 & 2.2 \\
\hline La Unión & 3 & 1.3 \\
\hline Resto del departamento & 95 & 42.4 \\
\hline Cobertura en el departamento $(\%)$ & & $100 \%$ \\
\hline
\end{tabular}

Fuente: Ministerio de Salud y la Protección Social

que se presenta concentración de los servicios en las áreas urbanas de tres municipios. Esto hace que se constituya en un recurso con una barrera en el acceso geográfico y físico, por la ubicación de los prestadores y la disponibilidad del servicio, y al mismo tiempo en una barrera de tipo económico.

En cuanto a la atención por especialidades de odontología, se encontró que en el sector público se cuenta con la atención por parte de talento humano con especialización en odontología en los municipios de Pasto, Ipiales y Ricaurte, así:

El municipio de Ricaurte cuenta con el primer nivel de atención en salud que corresponde a una IPS indígena y a una ESE. Los servicios de especialidades prestados en este municipio son: rehabilitación oral, periodoncia, ortodoncia y endodoncia, los cuales están a cargo de la ESE. Este municipio se encuentra a $142 \mathrm{~km}$ de la ciudad de Pasto, el tiempo de viaje en automóvil es cercano a las 3 horas.

El municipio de Ipiales, con segundo nivel de atención en salud, presta los servicios de las especialidades de cirugía oral y maxilofacial, endodoncia, periodoncia y odontopediatría. Este municipio se encuentra a 1 hora y 30 minutos de Pasto.

El municipio de La Unión presta el servicio de odontopediatría en el hospital de segundo nivel con el que cuenta; los hospitales del mismo nivel de los municipios de Pasto y Tumaco no registran en la base de datos del Ministerio la atención por odontología en ninguna especialidad, ni tampoco por odontología general. El único hospital público de tercer nivel del departamento no tiene registrada en la base de datos del Ministerio la atención por parte de las especialidades, ni por odontología general, solamente se encuentra registrada la atención por cirugía oral y cirugía maxilofacial, el resto de especialidades se concentran en los municipios de Ipiales y Ricaurte.

En cuanto al sector privado de atención en salud oral, se puede apreciar que existen municipios que no cuentan con ninguna atención especializada de odontología. Los usuarios, por consiguiente, deben desplazarse a donde se cuente con este tipo de servicios, que se concentran en pocos municipios. Es evidente 
que existe una alta concentración de servicios en la ciudad de Pasto, con un total de 139 prestadores, y el número presente en el departamento es de 174 , es decir, que el $80 \%$ de los especialistas están ubicados en la capital. Predominan los especialistas en ortodoncia, con un número total departamental de 63, seguido de endodoncia, rehabilitación oral, odontopediatría, periodoncia, cirugía oral y cirugía maxilofacial, en menor medida. Esta concentración en la capital, además del bajo número de especialistas en áreas diferentes a la ortodoncia, hace que la odontología especializada tenga una baja disponibilidad para la población y representa tiempo de viaje y desplazamiento para quienes requieran este tipo de atención. Por ello, es un servicio con barrera de acceso geográfica para la mayoría de los municipios del departamento.

La ciudad de Pasto es el único municipio en el que se cuenta con todas las especialidades de la odontología en el sector privado, pues en el resto del departamento hay municipios que no cuentan con ningún especialista en ninguna rama de la odontología; solo 11 municipios de los 64 prestan al menos un servicio de odontología especializada y en su mayoría corresponde a la ortodoncia.

\section{Inventario vial de Nariño en relación con los prestadores de salud}

El transporte en el territorio colombiano está regido por la Ley 105 de 1993. Esta norma dicta que el Estado es el encargado de la planeación, el control, la regulación y la vigilancia de las actividades vinculadas; además, debe proveer y velar por la seguridad ciudadana, ya que el transporte es el pilar del desarrollo del país y de la unidad nacional. Las vías de comunicación deben incentivar la integración regional, para de esta forma poder promover el desarrollo social de la población en cuanto a salud, recreación y educación. En este marco, el trasporte terrestre es el más usado en los departamentos de Colombia y este debe responder a las necesidades y requerimientos de desplazamientos de la población, en condiciones de accesibilidad, comodidad, calidad y seguridad $(7,38,39)$.

El departamento de Nariño, por su ubicación y características geográficas, presenta una red vial compleja que lo conecta tanto con la capital San Juan de Pasto como con el resto del país y con la República del Ecuador. Sus vías se encuentran en terreno pavimentado y afirmado, con un total aproximado de $6500 \mathrm{~km}$ de carreteras. De estos, $3922 \mathrm{~km}$ se encuentran en terreno afirmado y 1213 km pavimentados; los kilómetros de vías restantes se encuentran en mal estado o son inexistentes. La mayoría de las vías se concentran en la zona andina y existe una evidente escasez de vías en la zona del Pacífico $(40,41)$.

De los $6500 \mathrm{~km}$ de red vial del departamento de Nariño, $653.79 \mathrm{~km}$ pertenecen a la red troncal y transversal, es decir, son carreteras primarias o también denominadas nacionales, de los cuales el $80.7 \%$ es pavimentado y el $19.3 \%$ se encuentra en terreno afirmado; estas carreteras comunican el departamento de sur a norte y de oriente a occidente. La red secundaria la constituyen aproximadamente $1605 \mathrm{~km}$, de los cuales el 7\% se encuentra pavimentado y el $93 \%$ en terreno afirmado. De estos, el $1.9 \%$ se encuentra en estado óptimo, el $57 \%$ en estado regular y el $41 \%$ en mal estado; estas vías conectan al departamento de forma intermunicipal y rural. Las vías terciarias corresponden a $1508 \mathrm{~km}$, y $2736 \mathrm{~km}$, que pertenecen a los municipios, son vías que conectan las cabeceras municipales, los corregimientos y las veredas (42).

A efectos de la investigación, se hizo una medida de la distancia de cada municipio a la ciudad de Pasto, puesto que es la única que tiene todos los niveles de atención, tanto públicos como privados, y además tiene talento humano capacitado en salud oral de todas las especialidades (tabla 6). 


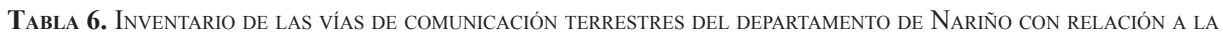
Distancia hasta Pasto y estado PaVimentado o AFIRMAdo, 2013

\begin{tabular}{|c|c|c|c|}
\hline Municipio & $\begin{array}{c}\text { Distancia en km hasta } \\
\text { Pasto }\end{array}$ & $\begin{array}{c}\text { Longitud pavimento } \\
(\mathbf{k m})\end{array}$ & $\begin{array}{c}\text { longitud afirmado } \\
(\mathrm{km})\end{array}$ \\
\hline Albán & 68 & 37 & 31 \\
\hline Aldana & 92 & 87 & 5 \\
\hline Ancuya & 83 & 41 & 42 \\
\hline Arboleda & 72 & 53 & 19 \\
\hline Barbacoas & 235 & 196.7 & 38.3 \\
\hline Bélen & 89 & 37 & 52 \\
\hline Buesaco & 37 & 37 & 0 \\
\hline Chachagui & 29 & 29 & 0 \\
\hline Colón & 127 & 87 & 40 \\
\hline Consaca & 55 & 55 & 0 \\
\hline Córdoba & 90 & 75 & 15 \\
\hline Cotadero & 68 & 65 & 3 \\
\hline Cuaspud Carlosama & 93 & 85 & 8 \\
\hline Cumbal & 123 & 108.1 & 19.9 \\
\hline Cumbitara & 164 & 84.2 & 79.8 \\
\hline El Charco & 465 & NA & NA \\
\hline El Peñol & 52 & 16 & 36 \\
\hline El Rosario & 124 & 106.7 & 17.3 \\
\hline El Tablón & 62 & 37 & 22 \\
\hline El Tambo & 36 & 16 & 20 \\
\hline Francisco Pizarro & 442 & NA & NA \\
\hline Funes & 50 & 48 & 2 \\
\hline Guachucal & 92 & 69 & 23 \\
\hline Guaitarilla & 75 & 75 & 0 \\
\hline Gualmatan & 86 & 78.5 & 7.5 \\
\hline Iles & 65 & 43 & 22 \\
\hline Imues & 52 & 52 & 0 \\
\hline Ipiales & 80 & 80 & 0 \\
\hline La Cruz & 103 & 54 & 49 \\
\hline La Florida & 7 & 7 & 0 \\
\hline La Llanada & 140 & 13 & 127 \\
\hline La Tola & 460 & NA & NA \\
\hline La Unión & 92 & 87 & 5 \\
\hline Leiva & 185 & 137 & 48 \\
\hline Linares & 90 & 16 & 74 \\
\hline Los Andes & 69 & 16 & 53 \\
\hline
\end{tabular}




\begin{tabular}{|c|c|c|c|}
\hline Municipio & $\begin{array}{c}\text { Distancia en km hasta } \\
\text { Pasto }\end{array}$ & $\begin{array}{c}\text { Longitud pavimento } \\
\text { (km) }\end{array}$ & $\begin{array}{c}\text { longitud afirmado } \\
(\mathbf{k m})\end{array}$ \\
\hline Magui Payán & 380 & 196.7 & 55.3 \\
\hline Mallama & 121 & 121 & 0 \\
\hline Mosquera & 513 & NA & NA \\
\hline Nariño & 20 & 20 & 0 \\
\hline Olaya Herrera & 70 & NA & NA \\
\hline Ospina & 75 & 64 & 11 \\
\hline Policarpa & 92 & 49.7 & 42.3 \\
\hline Potosí & 96 & 96 & 0 \\
\hline Providencia & 85 & 52 & 33 \\
\hline Puerres & 80 & 78.4 & 1.6 \\
\hline Pupiales & 94 & 85 & 9 \\
\hline Ricaurte & 142 & 142 & 0 \\
\hline Roberto Payán & 254 & NA & NA \\
\hline Samaniego & 118 & 118 & 0 \\
\hline San Bernardo & 75 & 53 & 22 \\
\hline San Lorenzo & 66 & 34 & 32 \\
\hline San Pablo & 133 & 54 & 65 \\
\hline San Pedro de Cartago & 84 & 54 & 30 \\
\hline Sandoná & 48 & 41 & 7 \\
\hline $\begin{array}{l}\text { Santa Bárbara (Iscuan- } \\
\text { de) }\end{array}$ & 550 & NA & NA \\
\hline Santacruz & 108 & 52 & 56 \\
\hline Sapuyes & 78 & 72 & 6 \\
\hline Taminango & 86 & 65 & 21 \\
\hline Tangua & 28 & 28 & 0 \\
\hline Tumaco & 284 & 284 & 0 \\
\hline Túquerres & 72 & 72 & 0 \\
\hline Yacuanquer & 25 & 25 & 0 \\
\hline
\end{tabular}

Fuente: Secretaría de Infraestructura y Minas de la Gobernación de Nariño

En Nariño predominan las vías con terreno pavimentado, pero en un estado de regular a malo (42). Esto hace que el desplazamiento entre regiones requiera un mayor tiempo del estimado, o que no se comuniquen con el departamento, y sea necesario desplazarse a otro departamento.

Hay municipios que están incomunicados por vía terrestre. La población de la zona del Pacífico debe acudir al transporte fluvial, que eleva costos de transporte; además, el transporte de pasajeros por este medio no es el más adecuado y presenta falencias en los equipos que se utilizan.

A partir del inventario de las vías del departamento, en la tabla 7 se recogen los datos del tiempo de viaje. Se incluyen los 64 municipios del departamento, el tiempo en horas que 
tarda desplazarse de un municipio a la capital y los medios de trasporte que se pueden usar.

La barrera de acceso geográfica para la población que se desplaza caminando hasta la ciudad de Pasto, con el objetivo de acceder a un siguiente nivel de atención, está presente en 61 municipios de Nariño. No se presenta en dos municipios (La Florida y Nariño), pues cumplen con los dos determinantes del tiempo de viaje y la distancia (figura 3).

En el desplazamiento en caballo, se evidenció que solamente 2 municipios (La Florida y Na-

Tabla 7. Resultado de modelo de distancia-tiempo: tiempo de Viaje, Para el DesPlazamiento en El departamento de Nariño hasta San Juan de Pasto, 2012

\begin{tabular}{|c|c|c|c|c|c|c|}
\hline \multicolumn{7}{|c|}{ Distancia y tiempo de desplazamiento en diferentes medios de transporte en el departamento de Nariño } \\
\hline \multirow[b]{2}{*}{ Municipio } & \multirow[b]{2}{*}{$\begin{array}{l}\text { Distancia } \\
\text { en km has- } \\
\text { ta Pasto }\end{array}$} & \multicolumn{5}{|c|}{ Tiempo en horas } \\
\hline & & Bicicleta & Carro & Caminando & Caballo & $\begin{array}{l}\text { Lancha } \\
\text { rápida }\end{array}$ \\
\hline Albán & 68 & 6.8 & 1.24 & 13.6 & 11.3 & \\
\hline Aldana & 92 & 9.2 & 1.67 & 18.4 & 15.3 & \\
\hline Ancuya & 83 & 8.3 & 1.51 & 16.6 & 13.8 & \\
\hline Arboleda & 72 & 7.2 & 1.31 & 14.4 & 12.0 & \\
\hline Barbacoas & 235 & 23.5 & 4.27 & 47 & 39.2 & \\
\hline Bélen & 89 & 8.9 & 1.62 & 17.8 & 14.8 & \\
\hline Buesaco & 37 & 3.7 & 0.67 & 7.4 & 6.2 & \\
\hline Chachagui & 29 & 2.9 & 0.53 & 5.8 & 4.8 & \\
\hline Colón & 127 & 12.7 & 2.31 & 25.4 & 21.2 & \\
\hline Consaca & 55 & 5.5 & 1 & 11 & 9.2 & \\
\hline Córdoba & 90 & 9 & 1.64 & 18 & 15.0 & \\
\hline Cotadero & 68 & 6.8 & 1.24 & 13.6 & 11.3 & \\
\hline Cuaspud Carlosama & 93 & 9.3 & 1.69 & 18.6 & 15.5 & \\
\hline Cumbal & 123 & 12.3 & 2.24 & 24.6 & 20.5 & \\
\hline Cumbitara & 164 & 16.4 & 2.98 & 32.8 & 27.3 & \\
\hline El Charco & 465 & NA & NA & NA & NA & $\begin{array}{c}5 \text { horas de } \\
\text { Tumaco/1 } \\
\text { hora de } \\
\text { Guapi }\end{array}$ \\
\hline El Peñol & 52 & 5.2 & 0.95 & 10.4 & 8.7 & \\
\hline El Rosario & 124 & 12.4 & 2.25 & 24.8 & 20.7 & \\
\hline El Tablón & 62 & 6.2 & 1.13 & 12.4 & 10.3 & \\
\hline El Tambo & 36 & 3.6 & 0.65 & 7.2 & 6.0 & \\
\hline Francisco Pizarro & 442 & NA & NA & NA & NA & \\
\hline Funes & 50 & 5 & 0.91 & 10 & 8.3 & \\
\hline Guachucal & 92 & 9.2 & 1.67 & 18.4 & 15.3 & \\
\hline Guaitarilla & 75 & 7.5 & 1.36 & 15 & 12.5 & \\
\hline Gualmatán & 86 & 8.6 & 1.56 & 17.2 & 14.3 & \\
\hline Iles & 65 & 6.5 & 1.18 & 13 & 10.8 & \\
\hline Imues & 52 & 5.2 & 0.95 & 10.4 & 8.7 & \\
\hline
\end{tabular}




\begin{tabular}{|c|c|c|c|c|c|c|}
\hline \multicolumn{7}{|c|}{ Distancia y tiempo de desplazamiento en diferentes medios de transporte en el departamento de Nariño } \\
\hline \multirow[b]{2}{*}{ Municipio } & \multirow[b]{2}{*}{$\begin{array}{c}\text { Distancia } \\
\text { en km has- } \\
\text { ta Pasto }\end{array}$} & \multicolumn{5}{|c|}{ Tiempo en horas } \\
\hline & & Bicicleta & Carro & Caminando & Caballo & $\begin{array}{c}\text { Lancha } \\
\text { rápida }\end{array}$ \\
\hline Ipiales & 80 & 8 & 1.45 & 16 & 13.3 & \\
\hline La Cruz & 103 & 10.3 & 1.87 & 20.6 & 17.2 & \\
\hline La Florida & 7 & 0.7 & 0.13 & 1.4 & 1.2 & \\
\hline La Llanada & 140 & 14 & 2.55 & 28 & 23.3 & \\
\hline La Tola & 460 & NA & NA & NA & NA & \\
\hline La Unión & 92 & 9.2 & 1.67 & 18.4 & 15.3 & \\
\hline Leiva & 185 & 18.5 & 3.36 & 37 & 30.8 & \\
\hline Linares & 90 & 9 & 1.64 & 18 & 15.0 & \\
\hline Los Andes & 69 & 6.9 & 1.25 & 13.8 & 11.5 & \\
\hline Magui Payán & 380 & 38 & 6.91 & 76 & 63.3 & \\
\hline Mallama & 121 & 12.1 & 2.2 & 24.2 & 20.2 & \\
\hline Mosquera & 513 & NA & NA & NA & NA & \\
\hline Nariño & 20 & 2 & 0.36 & 4 & 3.3 & \\
\hline Olaya Herrera & 70 & NA & NA & NA & NA & \\
\hline Ospina & 75 & 7.5 & 1.36 & 15 & 12.5 & \\
\hline Policarpa & 92 & 9.2 & 1.67 & 18.4 & 15.3 & \\
\hline Potosí & 96 & 9.6 & 1.75 & 19.2 & 16.0 & \\
\hline Providencia & 85 & 8.5 & 1.55 & 17 & 14.2 & \\
\hline Puerres & 80 & 8 & 1.45 & 16 & 13.3 & \\
\hline Pupiales & 94 & 9.4 & 1.71 & 18.8 & 15.7 & \\
\hline Ricaurte & 142 & 14.2 & 2.58 & 28.4 & 23.7 & \\
\hline Roberto Payán & 254 & NA & NA & NA & NA & \\
\hline Samaniego & 118 & 11.8 & 2.15 & 23.6 & 19.7 & \\
\hline San Bernardo & 75 & 7.5 & 1.36 & 15 & 12.5 & \\
\hline San Lorenzo & 66 & 6.6 & 1.2 & 13.2 & 11.0 & \\
\hline San Pablo & 133 & 13.3 & 2.42 & 26.6 & 22.2 & \\
\hline San Pedro de Cartago & 84 & 8.4 & 1.53 & 16.8 & 14.0 & \\
\hline Sandoná & 48 & 4.8 & 0.87 & 9.6 & 8.0 & \\
\hline Santa Bárbara (Iscuande) & 550 & NA & NA & NA & NA & \\
\hline Santacruz & 108 & 10.8 & 1.96 & 21.6 & 18.0 & \\
\hline Sapuyes & 78 & 7.8 & 1.42 & 15.6 & 13.0 & \\
\hline Taminango & 86 & 8.6 & 1.56 & 17.2 & 14.3 & \\
\hline Tangua & 28 & 2.8 & 0.51 & 5.6 & 4.7 & \\
\hline Tumaco & 284 & 28.4 & 5.16 & 56.8 & 47.3 & \\
\hline Túquerres & 72 & 7.2 & 1.31 & 14.4 & 12.0 & \\
\hline Yacuanquer & 25 & 2.5 & 0.45 & 5 & 4.2 & \\
\hline
\end{tabular}


Figura 3. Tiempo de Viaje CAminando hasta la capital Pasto, CiUdad Con tercer nivel de atención

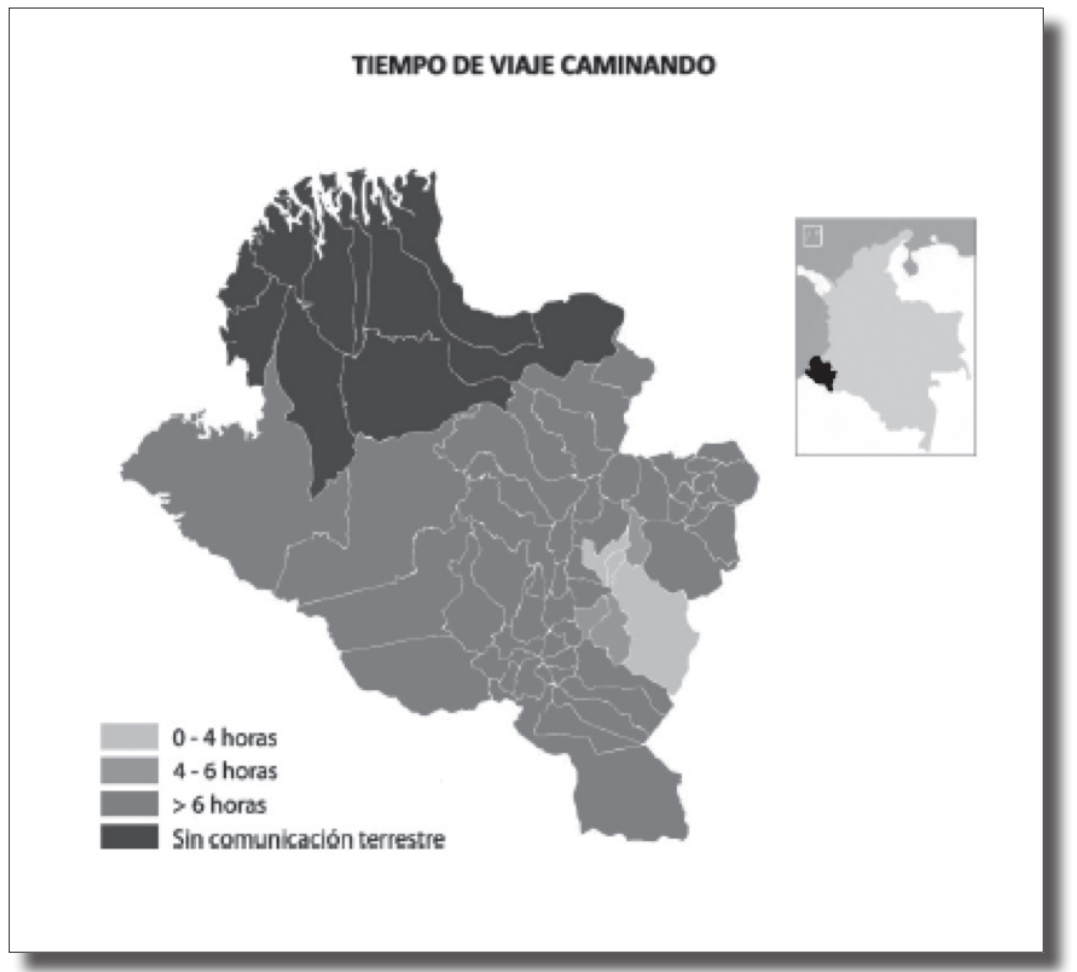

Fuente: elaboración propia

riño) se encuentran con los dos determinantes para que no exista barrera de acceso geográfica (4 horas de viaje y $25 \mathrm{~km}$ de distancia) al siguiente nivel de atención; es decir, que el resto del departamento tiene barrera de acceso geográfico a salud cuando se usa este medio de transporte como único para lograr el desplazamiento.

En el departamento de Nariño, el transporte en caballo es común, más todavía en las zonas rurales. Este medio de transporte tiene un alto impacto y a la vez conlleva limitaciones en el acceso a la salud, puesto que requiere un tiempo de viaje elevado, que además se ve alterado por el manejo del animal y su tiempo de recuperación, pues no se puede forzar a caminar al caballo por un tiempo mayor a 6 horas.

Se encontró que los habitantes de algunos municipios se desplazan en bicicleta dentro del departamento para llegar a Pasto. Son 7 los municipios que están dentro del rango de las 4 horas de viaje, es decir, que teóricamente no tienen barrera de acceso a los niveles de atención más altos, pero no todos cumplen con el otro requisito para determinar la existencia de barrera de acceso $(25 \mathrm{~km}$ de distancia al centro de atención del siguiente nivel). Con este límite se encuentran tres municipios (Yacuanquer, Nariño y La Florida), que cumplen con los dos requisitos. En el resto del departamento hay barrera de acceso geográfico, es decir, que 56 municipios del departamento de Nariño no tienen acceso al segundo o al tercer nivel de atención en salud si usan la bicicleta como único medio de transporte (ver figura 4).

En cuanto al desplazamiento que la población debe hacer para llegar a un siguiente 
nivel de atención en salud en un vehículo tipo automóvil, se encontró que en el departamento de Nariño 53 municipios no presentan barrera de acceso en el determinante de 4 horas de tiempo de viaje hasta la ciudad de Pasto. En cuanto al determinante de barrera de acceso, hasta $25 \mathrm{~km}$ de distancia al siguiente nivel de atención, solo tres municipios no tienen esa barrera ( $\mathrm{La}$ Florida, Nariño y Yacuanquer); el resto del departamento la enfrenta.

Los municipios que se encuentran hacia la zona del Pacífico, como Barbacoas, Tumaco y Magüi Payán, presentan barrera de acceso geográfico a salud, puesto que se encuentran a más de 4 horas de viaje en automóvil. El tiempo empleado llega hasta las 7 horas y a una distancia de $380 \mathrm{~km}$ hasta Pasto. Los municipios del Pacífico (El Charco, Francisco Pizarro, La Tola, Mosquera, Olaya Herrera,
Roberto Payán y Santa Bárbara) se encuentran incomunicados por vía terrestre con el resto del departamento; además, su ubicación dentro del departamento está entre los 70 y los $550 \mathrm{~km}$ de distancia a la ciudad de Pasto. Las vías de comunicación en esa zona son fluviales y el transporte de pasajeros es limitado, pues la infraestructura no es adecuada y los pasajeros se ven obligados a usar los servicios disponibles, con las limitaciones existentes (figura 5).

\section{Proporción odontólogo/habitantes en el departamento de Nariño}

La OMS, con el objetivo de mejorar la calidad de vida de la población y preservar la salud, ha planteado desde los años sesenta que el número ideal de habitantes por odontólogo es de 3000/1(1969), y esto persistió hasta el

Figura 4. Tiempo de viaje en bicicleta hasta la capital Pasto, ciudad con tercer nivel de atención

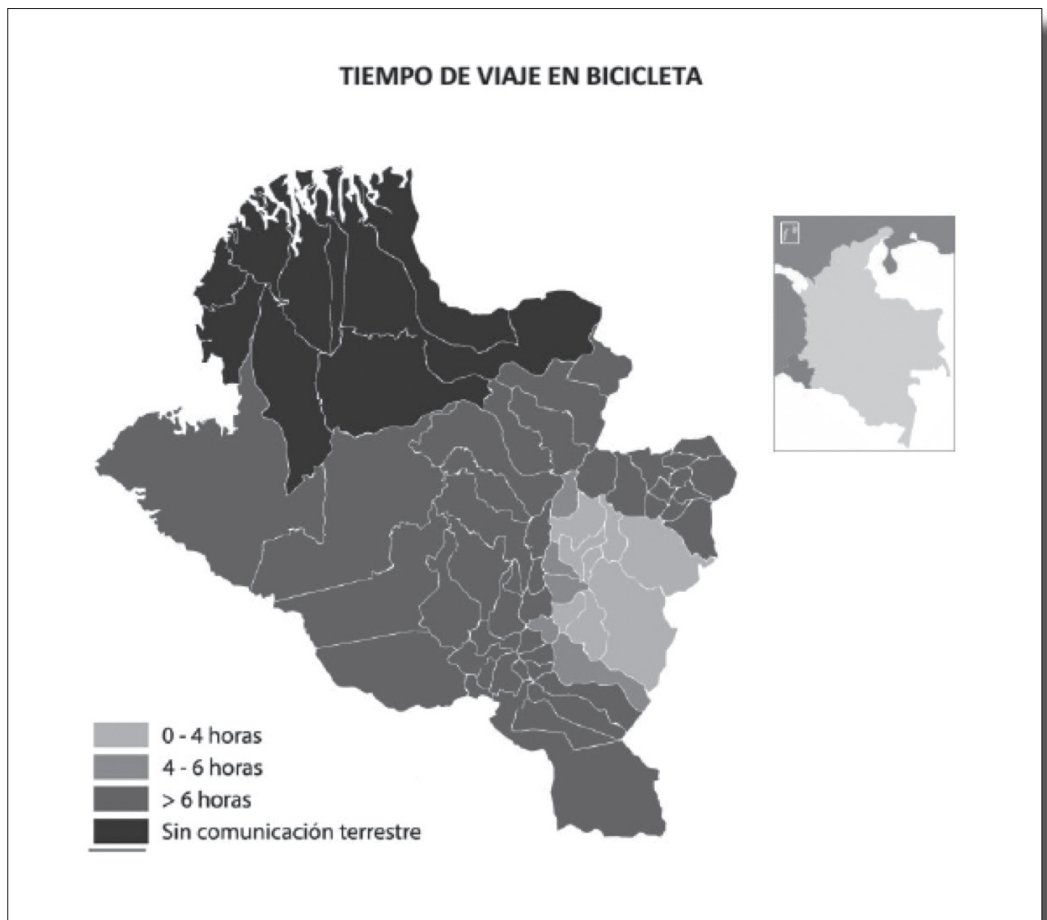

Fuente: elaboración propia. 
Figura 5. Tiempo de viaje en automóvil o carro hasta la capital Pasto, Ciudad con tercer nivel de atención

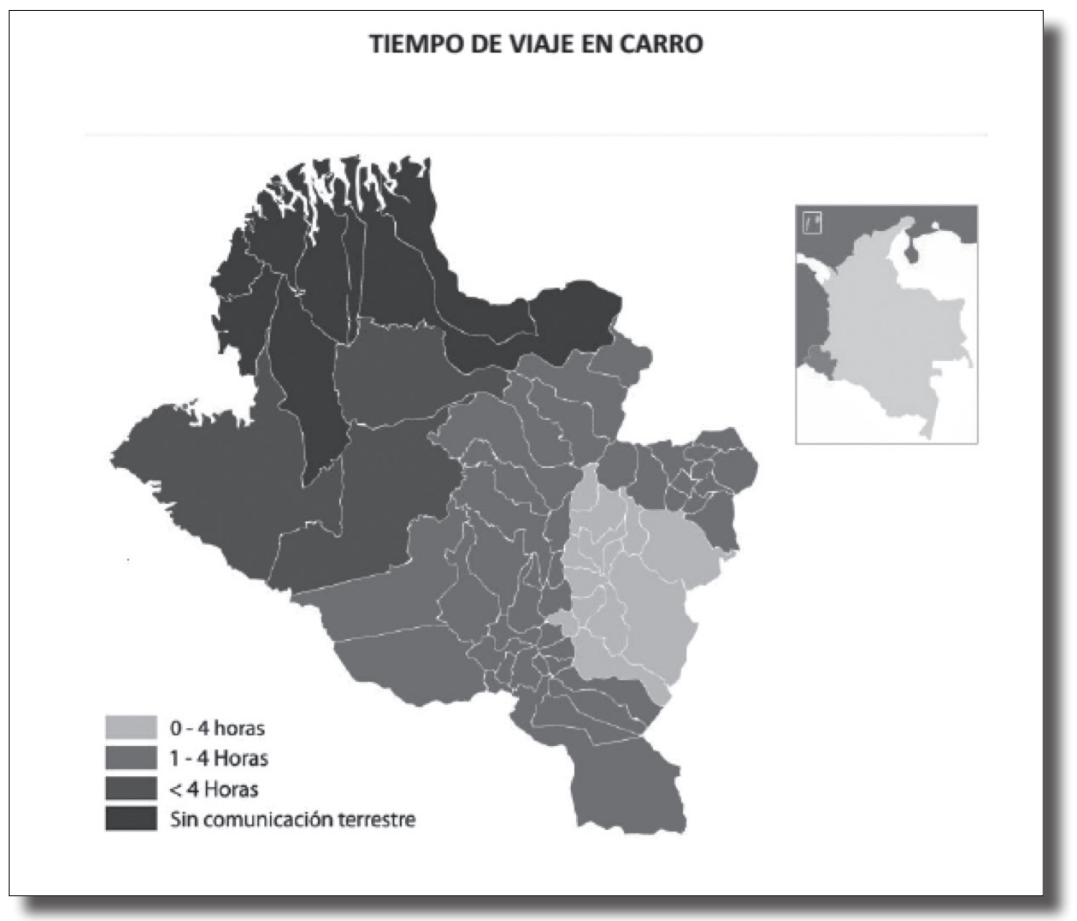

Fuente: elaboración propia

2010. Este es el valor para que la atención en salud oral sea calificada como aceptable para toda la población. Se recuerda a los lectores que esta investigación solamente indagó por el número y la distribución de los profesionales, y no por la compleja trama que significaría valorar la idoneidad de las instalaciones, así como las competencias profesionales de las personas vinculadas a los servicios, tema que desborda los alcances de este trabajo y amerita otra investigación.

Una cifra cercana a esta meta se logró en Colombia para el año 1987, donde la proporción era de 3500:1, lo que rápidamente cambio a 2500 personas por odontólogo para 1989. Este drástico cambio se logró debido a la creación de nuevas facultades de odontología en el país, el ingreso de estas por la educación privada y un creciente número de profesionales en salud oral que rápidamente logró elevar la dinámica de la proporción odontólogo-paciente (43).

En cuanto al número de odontólogos por habitantes, cada país es el encargado de determinar sus propios objetivos, teniendo en cuenta las necesidades de salud oral y al mismo tiempo regular y mantener un adecuado equilibrio, ajustando con los años la proporción de forma gradual, para así conseguir una asistencia efectiva (44).

Se encontró que en el departamento de Nariño existen 3 (de 64) municipios con una relación adecuada de odontólogo por habitantes (Roberto Payán, Francisco Pizarro y Chachagui), tomando como valor base el proporcionado por la OMS (alrededor de los 3000 habitantes por cada odontólogo), lo cual significa que la disponibilidad del servicio es adecuada. Se evidenció sobreoferta del servicio de odontología en 4 municipios del 
departamento de Nariño (Magui, La Tola, Cumbal y Barbacoas), puesto que el número de habitantes por odontólogos es menor a 3000. En general, el departamento presenta una baja oferta del servicio de odontología, puesto que la proporción general es de 6700 habitantes por odontólogo.

En Nariño, 20 municipios tienen una proporción mayor a los 10000 habitantes por odontólogo, de los cuales 2 (El Charco y Sandoná) presentan una relación mayor a los 20000 habitantes. Estos datos permiten observar que donde aparentemente existe el servicio de odontología, este no logra dar cubrimiento a toda la población, puesto que la oferta del servicio es limitada y por tanto la disponibilidad se presenta como una barrera de acceso.

\section{Discusión y conclusiones}

Esta investigación partió de la pregunta por las barreras geográficas de acceso a los diferentes niveles de atención en salud en el departamento de Nariño, así como de la pregunta sobre la presencia y distribución de los recursos humanos en odontología general y especializada en el departamento.

Los niveles de atención en salud del sector público y del sector privado en el departamento de Nariño es necesario analizarlos individualmente, dada su desigual distribución geográfica en el interior del departamento y en los municipios. Fue así como se identificó que el primer nivel de complejidad de atención en salud del sector público está presente en todo el departamento y cubre al $100 \%$ de los municipios, con al menos un centro de atención en salud que presta los servicios contemplados en este nivel de atención. Esto significa que, teóricamente, hay una buena cobertura y se supondría que es asequible, disponible y accesible para toda la población.
El segundo nivel de complejidad en salud está representado por 4 prestadores, en tan solo 4 municipios (Pasto, La Unión, Ipiales y Tumaco), lo cual permite afirmar que esta es una distribución limitada para atender a las necesidades de todo el departamento, que además de ser numeroso en cantidad de municipios, presenta una geografía compleja (cordillera andina, valle interandino y corredor Pacífico), lo que en la práctica disminuye las posibilidades de llegar a este nivel de atención a casi la totalidad de la población del departamento.

El tercer nivel de atención en salud del sector público solo se encuentra en la ciudad de Pasto, con un prestador (Hospital Universitario Departamental de Nariño) que debe atender las necesidades a este nivel de todo el departamento. Este nivel de atención tiene barrera de acceso para el departamento, a excepción de los municipios que se encuentran dentro de la distancia de $25 \mathrm{~km}$ de la ciudad de Pasto y con un tiempo de viaje menor a 4 horas en cualquier medio de transporte (Nariño, La Florida y Yacuanquer). De lo anterior se puede inferir que los municipios donde la barrera de acceso es más acentuada están en la costa pacífica, puesto que el tiempo de viaje en cualquier medio de transporte hasta Pasto es mayor a 4 horas, llegando incluso a superar las 7 horas por trayecto.

En relación con los servicios en el sector privado, se encontró que la complejidad baja está centralizada en la capital y en los municipios de Tumaco e Ipiales; el resto carece de cobertura de este nivel y se encuentra supeditado a la oferta del sector público. Una situación agravante relacionada con estos prestadores privados es que a pesar de compartir la baja complejidad, no brindan servicios unificados, como sí lo hace el sector público, pues en ocasiones se concentran en un solo servicio, regido por las leyes de la ganancia, y en ocasiones puede tratarse de 
profesionales independientes, lo cual contribuye a la poca oferta de servicios a este nivel en la mayor parte del departamento.

El nivel de complejidad media del sector privado presenta un mayor número de prestadores al compararlo con el público, sin embargo, no alcanza a cubrir a toda la población. Una situación similar se encuentra en el nivel de complejidad alto, con un total de 34 prestadores, de los cuales 29 se concentran en la ciudad de Pasto, 3 en Ipiales y 2 en Tumaco. Este nivel de complejidad tiene un mayor número de prestadores de servicio que en el sector público, sin embargo, no puede suplir las necesidades de salud para toda la población, debido a la concentración en la capital del departamento.

Se encuentra que los odontólogos generales del sector público, que corresponden al primer nivel de atención en salud del departamento, están presentes en los 64 municipios, lo que conlleva el supuesto de que toda la población tiene acceso a un odontólogo general y a los procedimientos en salud oral que corresponden a ese nivel de atención. Teóricamente, estos hallazgos permitirían afirmar que el primer nivel de atención en odontología es un servicio de salud sin barrera de acceso y con disponibilidad para toda la población, pero cuando se hace la relación de odontólogo por cada 3000 habitantes, según la recomendación de la OMS (43), casi la totalidad de los municipios tiene una relación desfavorable.

En cuanto a los profesionales de odontología especializada, que corresponden al segundo y al tercer nivel de complejidad, es evidente que en el sector público son limitados, puesto que existen solamente 4 instituciones que registran la atención por parte de las especialidades de: endodoncia, ortodoncia, odontopediatría, rehabilitación oral y periodoncia, y no se presentan simultáneamente.
Se hizo evidente en la investigación que el haber trasladado la especialidad de endodoncia al segundo nivel de atención, puede presentar en el futuro efectos nocivos en la salud oral de la población aquejada por dolor de compromiso pulpar, ya que no todas las personas tienen el acceso o la capacidad de pago para acceder a este nivel de atención, lo cual podría generar poblaciones con edentulismo parcial o total en el curso de la próxima década.

Los profesionales especialistas en cirugía oral y cirugía maxilofacial se concentran exclusivamente en la ciudad de Pasto en el tercer nivel de atención. Esta distribución implica que los usuarios del sistema de salud público deben desplazarse hasta el prestador que les pueda ofrecer el servicio, lo que implica un tiempo de viaje y asumir el costo de uno o varios medios de transporte hasta los municipios que cuenten con la oferta del servicio.

En el sector privado, los especialistas en odontología se concentran en la ciudad de Pasto, donde se ubica un $80 \%$ del total del departamento; predominan la ortodoncia y la endodoncia, en tanto que las otras especialidades tienen menos del 10\% de representación cada una. De igual forma, se encuentran en un número bajo, que no logra suplir las necesidades de la población ni brindar una atención plena. Esta distribución de los prestadores especializados de odontología privados hace que los servicios presenten un limitado acceso, por la disponibilidad y la barrera de acceso geográfica, además del costo de la salud.

Es de destacar que se logró identificar la existencia de barreras de acceso de tipo geográfico en Nariño, con base en los determinantes de barrera de acceso de 4 horas de tiempo de viaje y $25 \mathrm{~km}$ de distancia. Es asî como se identificó que los recorridos hasta la 
ciudad de Pasto caminando y a caballo, para obtener atención en los niveles más altos, no son suficientes para que la población del departamento tenga acceso a la salud en un tiempo de viaje adecuado. El transporte en bicicleta no es un medio de transporte eficiente, orientado al objetivo de lograr una atención en salud en un tiempo adecuado, ya que solamente 7 municipios del departamento cumplen con los determinantes de acceso.

El desplazamiento dentro del departamento utilizando el automóvil como medio de transporte logra que la población tenga una posibilidad mayor de acceso a los prestadores de salud de los niveles más altos; sin embargo, no todos los municipios cumplen con el determinante de una distancia menor a 25 $\mathrm{km}$ de distancia al prestador. Se encontró que la zona de la llanura del Pacífico no cuenta con vías de comunicación terrestre.

El tiempo de viaje hasta el municipio de Tumaco oscila entre las 4 y las 6 horas, en donde se cuenta con un hospital de segundo nivel; sin embargo, cuando se requiere atención por el tercer nivel de complejidad, se debe hacer un desplazamiento de $284 \mathrm{~km}$ hasta la ciudad de Pasto, lo que implica un tiempo de viaje de 5 horas. Por esta razón, se encontró que en el caso de urgencias médicas, es más conveniente desplazarse al municipio de Buenaventura, donde hay más transporte fluvial que hacia el municipio de Tumaco, o viajar al Valle del Cauca o al Cauca en avión, para lograr una atención más rápida, lo que implica un menor tiempo de viaje y por ende una atención más rápida, pero un elevado costo en transporte.

A partir de los resultados obtenidos en este estudio se puede concluir que en el departamento de Nariño existen barreras geográficas de acceso a la salud, así como falta de disponibilidad de los prestadores de salud oral. Por lo tanto, el sistema de salud colombiano no está en la capacidad de responder a las necesidades de salud de la población y se encuentra en condiciones de garantizar el desarrollo pleno de la salud para la población.

Los académicos nos hemos pronunciado durante todos estos años de vigencia de la Ley 100 de 1993, en particular desde la declaratoria de la emergencia social, con respecto a la necesidad de una reforma estructural al sistema de salud —no ajustes ni mera cosmética-, ya que se ha documentado que hay un conflicto de valores, estructural y complejo, debido a la tensión entre los principios y los derechos establecidos en la Constitución de 1991, por un lado, y las leyes de mercado, con la búsqueda de rentabilidad financiera como fin fundamental, las prácticas clientelistas y el predominio del interés particular sobre el interés colectivo, por el otro.

La reforma a la que aspiramos parte de principios que han ido ganando el consenso de los más variados sectores. Entre estos principios se destacan: 1) la salud como derecho humano fundamental, vinculado a la ciudadanía o a la residencia, y no a la capacidad de pago diferenciada (cotización), como ha sido hasta ahora; 2) el Estado debe ser el garante de la regulación, la gestión y la gobernabilidad del sistema, reconociendo las necesidades diferenciadas por condiciones de vida, región geográfica, edad, género o estado de salud, privilegiando en esta regulación a los más vulnerados; 3) el concepto de salud debe ser entendido en su acepción más amplia, de manera que estén contempladas la alimentación saludable, el agua y el saneamiento ambiental, la vivienda digna y un empleo de calidad para toda(o)s, como bien fuera señalado por la Corte Constitucional en la Observación n. ${ }^{\circ} 14$ del 2001.

Claramente, en relación con estas propuestas, los grupos de interés al servicio de la acumulación del capital tratarán de imponer 
sus propuestas, con respecto a las cuales debemos desplegar toda nuestra imaginación para informar a la ciudadanía sobre las implicaciones de estas y otras reformas. Requerimos para ello una pedagogía ciudadana, ya no la del oprimido o la de la esperanza, como nos enseñara Paulo Freire en sus primeros escritos, sino una pedagogía que diga ibasta! Una pedagogía de la indignación — de nuevo retomando una de las últimas obras del pedagogo brasileño-que le hable con sentido y claramente a todas las personas, para aumentar el nivel de conciencia sobre la desfachatez del proyecto de mercantilización de la salud y garantizar la necesaria movilización que derrotará, como lo ha hecho en ocasiones anteriores, las pretensiones reaccionarias de una oligarquía que se niega a reconocer que ya no nos gobierna como en la época del absolutismo (45).

Movilización para hacer ver que la salud integral no es una mercancía exclusiva, de la que pueden disfrutar unos pocos; que ante el fracaso visible de la Ley 100 hay que oponer una reforma estructural consensuada con la mayor cantidad de actores sociales. Movilización para exigir a los legisladores que incluyan toda la evidencia que el movimiento social y la academia han aportado durante estos años y hacer públicos todos los debates y discusiones que afectarán uno de los valores más preciados por todos: el derecho a la salud.

\section{Referencias bibliográficas}

1. Organización Mundial de la Salud (OMS). Informe sobre la salud en el mundo, la financiación de los sistemas de salud: el camino hacia la cobertura universal. Ginebra, Suiza: OMS; 2010. p. 1-42.

2. Mauch V, Woods N, Kirubi B, Kipruto H, Sitienei J, Klinkenberg E. Assessing access barriers to tuberculosis care with the tool to estimate patients `cost: pilot results from two districts in Kenya. BMC Public Health. 2011;11(43):1-9

3. Apparicio P, Abdelmajid M, Riva M, et al. Comparing alternative approaches to measuring the geographical accessibility of urban health services: distance types and aggregation-error issues. Int $\mathbf{J}$ Health Geogr 2008;7(7): 33-8.

4. Guerrero E, Pan K, Curtis A, et al. Avaiability of substance abuse treatment services in Spanish: A GIS analysis of Latino communities in Los Angeles Country, California. Substance Abuse Treatment, Prevention and Policy. 2011;6(21):1-8.

5. Jordan H, Roderick P, Martín D, et al. Distance, rurality and the need for care: access to health services in South West England. Int J Health Geogr. 2004;3(21):1-9.

6. World Health Organization (WHO). Monitoring equity in access to AIDS treatment programmes: a review of concepts, models, methods and indicators Francia: WHO; 2010. p. 5-87

7. Colombia - Ministerio de Transporte, Oficina de Planeación. Caracterización del transporte en Colombia, diagnóstico y proyectos de transporte e infraestructura. Bogotá: Ministerio de Transporte; 2005. p. 15-97.

8. Grupo de Trabajo de la Comisión de Regulación en Salud (CRES). Evolución del Sistema General de Seguridad Social en Salud y recomendaciones para su mejoramiento. Informe anual para el Congreso de la república. Bogotá: CRES; 2010. p. 1-88.

9. Colombia - Congreso de la República. Ley 10 de 1990; Por la cual se reorganiza el Sistema Nacional de Salud y se dictan otras disposiciones. Bogotá: Congreso de la República; 1990.

10. Colombia - Congreso de la República. Decreto 1762 de 1990 por la cual se reglamenta parcialmente la ley 10 de 1990, en cuanto a la organización de los servicios de salud que se presentarán en los municipios del país. Bogotá: Congreso de la República; 1990.

11. Colombia - Congreso de la República. Ley 100 de 1993; Por la cual se crea el sistema de seguridad social integral y se dictan otras disposiciones. Bogotá: Congreso de la República; 1993.

12. Colombia - Ministerio de Salud. Resolución 5261 de 1994, por la cual se establece el manual de actividades, intervenciones y procedimientos del Plan Obligatorio de Salud del Sistema General de Seguridad Social en Colombia. Bogotá: Ministerio de Salud; 1994.

13. Colombia - Ministerio de Salud. Resolución 5261 de 1994; Por la cual se establece el manual de actividades, intervenciones y procedimientos del Plan Obligatorio de Salud en el Sistema General de Seguridad Social en Salud. Bogotá: Ministerio de Salud; 1994.

14. Colombia - Ministerio de Salud y Protección Social. Sede Web del Ministerio Salud y Protección Social [Internet]; 2012 [citado en mayo del 2012]. Disponible en: http://www.minsalud.gov.co/salud/ Paginas/INDICADORESBASICOSSP.aspx

15. Gobernación de Nariño. Sitio web de la Gobernación de Nariño [Internet]; 2012 [citado en mayo del 2012]. Disponible en: http://www.narino.gov. co/index.php/nuestro-departamento/informacion

16. Organización Mundial de la Salud (OMS) [Internet]; 2010 [citado en enero del 2013]. Disponible 
en: http://www.who.int/features/factfiles/universal_health_coverage/es/

17. Grupo de trabajo de la Comisión de Regulación en Salud (CRES). Evolución del Sistema General de Seguridad Social en Salud y recomendaciones para su mejoramiento. Informe anual para el Congreso de la República. Bogotá: CRES; 2011. p. 1-80.

18. Qidwai W, Ashfaq T, Khoja T, et al. Access to person-centered care a perspective on status, barriers, opportunities and challenges from the Eastern Mediterranean Region. Middel East Journal of Family Medicine. 2012;10(6):4-13

19. Guagliardo MF. Spatial accessibility of primary care: concepts, methods and challenges. Int $\mathbf{J}$ Health Geogr. 2004;3(3);1-13.

20. Comber A, Brunsdon C, Radbum R. A spatial analysis of variation in health access: linking geography, socio-economic status and access perception. Int J Health Geogr. 2011;10:44.

21. Bosanac E, Parkinson E, Hal D. Geographic access to hospice care: a 30 - minute travel time standard. Medical Care. 1976;14(7):616-24.

22. Wilson K, Rosenberg M. The geographies of crisis: exploring accesibility to health care in Canada. The Canadian Geographer. 2002;46(3):223-34.

23. Penchansky R, Thomas JW. "The Concept of Access: Definition and Relationship to Consumer Satisfaction." Medical Care 19, no. 2 (February 1981): 127-40.

24. Magilvy J, Congdon J. The crisis nature of health care transition for rural older adults. Public Health Nurs. 2000;17(5):336-45.

25. Rheuban KS. The role of telemedicine in fostering health - care innovation to addres problems of access, specialty shortages and changing patient care needs. Journal of Telemed and Telecare. 2006;12 Suppl 2:S45-50.

26. Bradley E, Thompson JW, Byam P, et al. Access and quality of rural heathcare: Ethiopian Millennium rural initiative. Int J Qual Health Care. 2011;23(3): 222-30.

27. Junqueira SR, Frias AC, Zilbovicius C, et al. Oral health and access to dental care services in relation to the health necessities index: Sao Paulo, Brazil, 2008. Cien and Saude Colet. 2012;17(4):1015-24.

28. Morrissey K, Clarke G, Ballas D, et al. Examining access to GP services in rural Ireland using microsimulation analysis. Royal Geographical Society. 2008;40(3):354-64.

29. Gao S, Mioc D, Anton F, et al. Online GIS services for mapping and sharing disease information. Int $\mathrm{J}$ Health Geogr. 2008;7:8.

30. Beyer KMN, Saftlas AF, Wallis AB, et al. A probabilistic sampling method (PSM) for estimating geographic distance to health services when only the region of residence is known. Int $\mathbf{J}$ Health Geogr.2011;10:4.

31. Calley LM. Using geographic information systems to desing population - based intervention. Public Health Nursing.2004;21(6):547-54.

32. Nuckols JR, Ward MH, Jarup L. Using Geographic information systems for exposure assessment in environmental epidemiology studies. Envirom Health Perspect. 2004;112(9):1007-15.

33. Colombia - Departamento Administrativo Nacional de Estadística (DANE). Censo General 2005, nivel nacional. Bogotá: DANE; 2005.

34. Instituto Nacional de Vías (Invias). Sitio web de INVIAS [Internet]; 2012 [citado en marzo del 2012]. Disponible en: http://www.invias.gov. co/index.php/historico-cartelera-virtual/doc_ download/845-direccion-territorial-nariño

35. Colombia - Gobernación de Nariño, Oficina de Planeación Departamental. Visión Nariño 2030 [Nariño 2030]. Pasto: Gobernación de Nariño; 2005.

36. Colombia - Ministerio de Tecnologías de la Información y las Comunicaciones. Vive Nariño un paraíso lleno de sorpresas [Internet]; 2013 [citado en febrero del 2013]. Disponible en: http://www. vivenarino.com/es/paraiso

37. Ortiz L, Cubides H, Restrepo D. Caracterización laboral del talento humano en salud de Colombia: Aproximaciones desde el ingreso base de cotización al Sistema General de Seguridad Social en Salud. Documento de trabajo. Bogotá: Ministerio de Salud y Protección Social Dirección de Desarrollo del Talento Humano en Salud República de Colombia.; 2012. p. 1-30.

38. Colombia, Constitución Política de 1991. Bogotá: Imprenta Nacional; 2015.

39. Colombia - Ministerio de Transporte. Ley 105 de 1993. Por la cual se dictan disposiciones básicas sobre el transporte, se distribuyen competencias y recursos entre la Nación y las entidades territoriales, se reglamenta la planeación en el sector transporte y se dictan otras disposiciones. Bogotá: Ministerio de Transporte; 1993.

40. Colombia - Ministerio de Obras Públicas y Transporte. Resolución 211425 de marzo de 1998. Bogotá: Ministerio de Obras Públicas y Transporte; 1998.

41. Viloria J. Documentos de trabajo sobre economía regional, economía del departamento de Nariño: ruralidad y aislamiento geográfico Cartagena: Banco de la República, Centro de Estudios Económicos Regionales; 2007.

42. Colombia - Gobernación de Nariño, Departamento Nacional de Planeación. Contrato Plan de la Nación con el departamento de Nariño, acuerdo estratégico para el desarrollo del territorio. Bogotá: Gobernación de Nariño, Departamento Nacional de Planeación; 2013.

43. Ternera J. Planificación de talento humano en salud oral en Colombia. Acta Odontol Col. 2011;1(1):77-92.

44. Organización Mundial de la Salud (OMS). La enseñanza de la odontología: informe de un comité de expertos en higiene oral. Ginebra: OMS; 1962.

45. Estrada-Montoya JH. La fiebre no está en las sábanas. A propósito del proyecto gubernamental de reforma a la salud. Revista Izquierda [Internet]. 2010 oct.; 5. Disponible desde: http://www.espaciocritico.com $/$ node $/ 58 \mathbf{x}$ 